\title{
Determination of matter potential from global analysis of neutrino oscillation data
}

\author{
M. C. Gonzalez-Garcia, ${ }^{a, b}$ Michele Maltoni, ${ }^{c}$ \\ ${ }^{a}$ C.N. Yang Institute for Theoretical Physics, State University of New York at Stony Brook, Stony \\ Brook, NY 11794-3840, USA \\ ${ }^{b}$ Institució Catalana de Recerca i Estudis Avançats (ICREA), Departament d'Estructura i Con- \\ stituents de la Matèria and Institut de Ciencies del Cosmos, Universitat de Barcelona, Diagonal \\ 647, E-08028 Barcelona, Spain \\ ${ }^{c}$ Instituto de Física Teórica UAM/CSIC, Calle de Nicolás Cabrera 13-15, Universidad Autónoma \\ de Madrid, Cantoblanco, E-28049 Madrid, Spain \\ E-mail: concha@insti.physics.sunysb.edu, michele.maltoni@csic.es
}

\begin{abstract}
We quantify our current knowledge of the size and flavor structure of the matter effects in the evolution of neutrinos based solely on the global analysis of oscillation neutrino data. The results are translated in terms of the present allowed ranges for the corresponding non-standard neutrino interactions in matter.
\end{abstract}

Keywords: Neutrino Physics, Solar and Atmospheric Neutrinos, Beyond Standard Model 


\section{Contents}

1 Introduction 1

2 Formalism 3

2.1 Earth matter potential for atmospheric and LBL neutrinos 4

$\begin{array}{lll}2.2 & \text { Earth matter potential for solar and KamLAND neutrinos } & 6\end{array}$

3 Analysis of solar and KamLAND data $\quad 7$

4 Results of global analysis $\quad 11$

$\begin{array}{lll}5 & \text { Summary } & 17\end{array}$

\section{Introduction}

It is now an established fact that neutrinos are massive and leptonic flavors are not symmetries of Nature [1,2]. This picture has now become fully proved thanks to the upcoming of a set of precise experiments which have confirmed the results obtained with solar and atmospheric neutrinos using terrestrial beams of neutrinos produced in nuclear reactors and accelerators facilities [3]. The minimum joint description of the neutrino data requires mixing among all the three known neutrinos $\left(\nu_{e}, \nu_{\mu}, \nu_{\tau}\right)$, which can be expressed as quantum superposition of three massive states $\nu_{i}(i=1,2,3)$ with masses $m_{i}$. Consequently when written in terms of mass eigenstates, the weak charged current interactions of leptons $[4,5]$ contain a leptonic mixing matrix which can be parametrized as:

$$
U_{\mathrm{vac}}=\left(\begin{array}{ccl}
c_{12} c_{13} & s_{12} c_{13} & s_{13} e^{-i \delta_{\mathrm{CP}}} \\
-s_{12} c_{23}-c_{12} s_{13} s_{23} e^{i \delta_{\mathrm{CP}}} & c_{12} c_{23}-s_{12} s_{13} s_{23} e^{i \delta_{\mathrm{CP}}} & c_{13} s_{23} \\
s_{12} s_{23}-c_{12} s_{13} c_{23} e^{i \delta_{\mathrm{CP}}} & -c_{12} s_{23}-s_{12} s_{13} c_{23} e^{i \delta_{\mathrm{CP}}} & c_{13} c_{23}
\end{array}\right),
$$

where $c_{i j} \equiv \cos \theta_{i j}$ and $s_{i j} \equiv \sin \theta_{i j}$. In addition to the Dirac-type phase $\delta_{\mathrm{CP}}$, analogous to that of the quark sector, there are two physical phases associated to the Majorana character of neutrinos, which are not relevant for neutrino oscillations $[6,7]$ and which are therefore omitted in the following.

In the simplest quantum-mechanical picture, flavor oscillations are generated by the kinematical Hamiltonian for this ensemble, $H_{\mathrm{vac}}$, which in the flavor basis $\left(\nu_{e}, \nu_{\mu}, \nu_{\tau}\right)$ reads

$$
H_{\mathrm{vac}}=U_{\mathrm{vac}} D_{\mathrm{vac}} U_{\mathrm{vac}}^{\dagger} \quad \text { with } \quad D_{\mathrm{vac}}=\frac{1}{2 E_{\nu}} \operatorname{diag}\left(0, \Delta m_{21}^{2}, \Delta m_{31}^{2}\right)
$$

The quantities $\Delta m_{21}^{2},\left|\Delta m_{31}^{2}\right|, \theta_{12}, \theta_{23}$, and $\theta_{13}$ are relatively well determined by the analysis of solar, atmospheric, reactor and accelerator experiments, while barely nothing is known on 
the $\mathrm{CP}$ phase $\delta_{\mathrm{CP}}$ and on the sign of $\Delta m_{31}^{2}$ [8-11]. Given the observed hierarchy between the solar and atmospheric mass-squared splittings there are two possible non-equivalent orderings for the mass eigenvalues, which are conventionally chosen as

$$
\begin{aligned}
& \Delta m_{21}^{2} \ll\left(\Delta m_{32}^{2} \simeq \Delta m_{31}^{2}\right) \text { with }\left(\Delta m_{31}^{2}>0\right) ; \\
& \Delta m_{21}^{2} \ll\left|\Delta m_{31}^{2} \simeq \Delta m_{32}^{2}\right| \text { with }\left(\Delta m_{31}^{2}<0\right) .
\end{aligned}
$$

As it is customary we refer to the first option, Eq. (1.3), as the normal ordering, and to the second one, Eq. (1.4), as the inverted ordering. Clearly they correspond to the two possible choices of the sign of $\Delta m_{31}^{2}$.

The flavor evolution of this neutrino ensemble is also affected by the difference in the matter potential induced by neutrino-matter interactions through the so-called MikheevSmirnov-Wolfenstein (MSW) mechanism [12, 13]. Within the context of the Standard Model (SM) of particle interactions, this effect is fully determined and leads to a matter potential which, for neutral matter, is proportional to the number density of electrons in the background $N_{e}(r), V=\sqrt{2} G_{F} N_{e}(r)$, and which only affects electron neutrinos. The evolution of the ensemble is then determined by the Hamiltonian $H^{\nu}=H_{\mathrm{vac}}+H_{\mathrm{mat}}^{\mathrm{SM}}$, with $H_{\mathrm{mat}}^{\mathrm{SM}}=\sqrt{2} G_{F} N_{e}(r) \operatorname{diag}(1,0,0)$. The magnitude and the presence of non-standard forms of the matter potential can be tested in solar neutrino experiments (and in combination with KamLAND) [14-27], as well and in the propagation of atmospheric and long-baseline neutrinos [28-54].

In this article we address our current knowledge of the size and flavor structure of the matter background effects in the evolution of solar, atmospheric, reactor and long-baseline (LBL) accelerator neutrinos based on the global analysis of oscillation data. To this aim, in Sec. 2 we briefly present the most general parametrization of the matter potential and its connection with non-standard neutrino interactions (NSI) in matter, which provide a well-known theoretical framework for this kind of phenomenological studies. We also discuss the simplifications used in the analysis of the solar+KamLAND sector and the atmospheric+LBL sector respectively. In Sec. 3 we present the results from the updated analysis of solar+KamLAND data and quantify the impact of the modified matter potential on the data description, as well as the status of the well-known "dark-side" solution which appears in presence of NSI. In Ref. [54] an analysis of atmospheric and LBL neutrino data was performed in the framework of a generalized matter potential, which extended the standard one by allowing for an arbitrary rescaling of the potential strength, a general rotation from the ee sector, and a rephasing with respect to $H_{\mathrm{vac}}$. It was concluded that the strength of the potential cannot be determined solely by these data, whereas its flavor composition is very much constrained. In Sec. 4 we update this analysis and revisit its conclusions after combining the results from atmospheric, LBL and reactor experiments with those from solar+KamLAND data. We show to what degree the determination of neutrino masses and mixing is robust even in the presence of this general form of the matter potential and we derive the most up-to-date allowed ranges on NSI parameters. Finally in Sec. 5 we summarize our results. 


\section{Formalism}

In the three-flavor oscillation picture, the neutrino evolution equation reads:

$$
i \frac{d}{d x}\left(\begin{array}{c}
\nu_{e} \\
\nu_{\mu} \\
\nu_{\tau}
\end{array}\right)=H^{\nu}\left(\begin{array}{c}
\nu_{e} \\
\nu_{\mu} \\
\nu_{\tau}
\end{array}\right)
$$

where $x$ is the coordinate along the neutrino trajectory and the Hamiltonian for neutrinos and antineutrinos is:

$$
H^{\nu}=H_{\mathrm{vac}}+H_{\mathrm{mat}} \quad \text { and } \quad H^{\bar{\nu}}=\left(H_{\mathrm{vac}}-H_{\mathrm{mat}}\right)^{*},
$$

with $H_{\text {vac }}$ given in Eq. (1.2). Thus the vacuum term has 6 parameters: $\Delta m_{21}^{2}, \Delta m_{31}^{2}, \theta_{12}$, $\theta_{13}, \theta_{23}, \delta_{\mathrm{CP}}$. In the Standard Model $H_{\text {mat }}$ is fully determined both in its strength and flavor structure to be $H_{\text {mat }}^{\mathrm{SM}}=\sqrt{2} G_{F} N_{e}(r) \operatorname{diag}(1,0,0)$ for ordinary matter. Generically ordinary matter is composed by electrons $(e)$, up-quarks $(u)$ and down-quarks $(d)$, thus in the most general case a non-standard matter potential can be parametrized as:

$$
H_{\text {mat }}=\sqrt{2} G_{F} N_{e}(r)\left(\begin{array}{ccc}
1 & 0 & 0 \\
0 & 0 & 0 \\
0 & 0 & 0
\end{array}\right)+\sqrt{2} G_{F} \sum_{f=e, u, d} N_{f}(r)\left(\begin{array}{ccc}
\varepsilon_{e e}^{f} & \varepsilon_{e \mu}^{f} & \varepsilon_{e \tau}^{f} \\
\varepsilon_{e \mu}^{f *} & \varepsilon_{\mu \mu}^{f} & \varepsilon_{\mu \tau}^{f} \\
\varepsilon_{e \tau}^{f *} & \varepsilon_{\mu \tau}^{f *} & \varepsilon_{\tau \tau}^{f}
\end{array}\right) .
$$

Since this matter term can be determined by oscillation experiments only up to an overall multiple of the identity, without loss of generality one can assume $\varepsilon_{\mu \mu}^{f}=0$. With this, we have 8 parameters (for each $f$ ) since $\varepsilon_{e e}^{f}$ and $\varepsilon_{\tau \tau}^{f}$ must be real whereas $\varepsilon_{e \mu}^{f}$, $\varepsilon_{e \tau}^{f}$ and $\varepsilon_{\mu \tau}^{f}$ can be complex.

In order to determine the relevant ranges for the parameters in the problem we must study which transformations leave the probabilities invariant. In particular we notice that any rephasing $H^{\nu} \rightarrow Q H Q^{*}$ where $Q=\operatorname{diag}\left(e^{i a}, e^{i b}, e^{i c}\right)$ leads to a rephasing of the scattering matrix $\exp \left(-i H^{\nu} L\right) \rightarrow Q \exp \left(-i H^{\nu} L\right) Q^{*}$, which does not affect the probabilities. In the standard oscillation scenario these symmetries are used to reduce the range of the mixing parameters, most commonly to $0 \leq \theta_{i j} \leq \pi / 2$ and $0 \leq \delta_{\mathrm{CP}} \leq 2 \pi$. In the presence of the non-standard matter potential they can be used just in the same way, thus reducing the range of the mixing parameters while keeping the phases of all the off-diagonal $\varepsilon_{\alpha \neq \beta}^{f}$. Alternatively, one could instead reduce the range for some of the $\varepsilon_{\alpha \neq \beta}^{f}$, at the price of retaining a wider range of the vacuum mixing angles. Furthermore, in the particular case of a unique $f$ and in the absence of the vacuum term it would be possible to use these symmetries to reduce the matter potential parameters from eight to six: two real flavor diagonal parameters, the absolute value of the flavor off-diagonal parameters, $\left|\varepsilon_{\alpha \neq \beta}^{f}\right|$, and one combination of their three complex phases, while the two additional phases would become unphysical. Only when both the vacuum term and the matter general potential are present the two additional phases become observable. Hence it is clear from this discussion that it is a matter of convention to include them in the matter potential or in the vacuum term. For real matter potential this means that only an overall sign of the three off-diagonal $\varepsilon_{\alpha \neq \beta}^{f}$ 
can be considered a generic feature of the matter potential, while the other two signs of $\varepsilon_{\alpha \neq \beta}^{f}$ can be traded off by enlarging the vacuum mixing parameters to $-\pi / 2 \leq \theta_{i j} \leq \pi / 2$. We will go back to this issue in the next section.

The standard theoretical framework for our proposed parametrization of the matter potential is provided by NSI affecting neutrino interactions in matter. They can be described by effective four-fermion operators of the form

$$
\mathcal{L}_{\mathrm{NSI}}=-2 \sqrt{2} G_{F} \varepsilon_{\alpha \beta}^{f P}\left(\bar{\nu}_{\alpha} \gamma^{\mu} \nu_{\beta}\right)\left(\bar{f} \gamma_{\mu} P f\right)
$$

where $f$ is a charged fermion, $P=(L, R)$ and $\varepsilon_{\alpha \beta}^{f P}$ are dimensionless parameters encoding the deviation from standard interactions. NSI enter in neutrino propagation only through the vector couplings so the induced matter Hamiltonian takes the form (2.3) with $\varepsilon_{\alpha \beta}^{f}=$ $\varepsilon_{\alpha \beta}^{f L}+\varepsilon_{\alpha \beta}^{f R}$.

\subsection{Earth matter potential for atmospheric and LBL neutrinos}

As seen above, in principle a generalized potential involves different parameters for the different charged fermions $f=e, u, d$ in the matter. In practice, however, for the propagation of atmospheric and LBL neutrinos the neutron/electron ratio $Y_{n}$ is reasonably constant all over the Earth. This implies that neutrino atmospheric and LBL oscillations are only sensitive to the sum of these interactions, weighted with the relative abundance of each particle. We can therefore define:

$$
\varepsilon_{\alpha \beta} \equiv \sum_{f=e, u, d}\left\langle\frac{Y_{f}}{Y_{e}}\right\rangle \varepsilon_{\alpha \beta}^{f}=\varepsilon_{\alpha \beta}^{e}+Y_{u} \varepsilon_{\alpha \beta}^{u}+Y_{d} \varepsilon_{\alpha \beta}^{d}
$$

The PREM model [55] fixes $Y_{n}=1.012$ in the Mantle and $Y_{n}=1.137$ in the Core, with an average value $Y_{n}=1.051$ all over the Earth. Since a proton has 2 up-quarks and 1 down-quark, a neutron has 1 up-quark and 2 down-quarks, and neutral matter obviously has the same number of protons and electrons $\left(Y_{p}=1\right)$, we get $Y_{u}=2+Y_{n}=3.051$ and $Y_{d}=1+2 Y_{n}=3.102$ in the Earth. With this in mind, the matter part of the Hamiltonian can be written as:

$$
H_{\mathrm{mat}}=\sqrt{2} G_{F} N_{e}(r)\left(\begin{array}{ccc}
1+\varepsilon_{e e} & \varepsilon_{e \mu} & \varepsilon_{e \tau} \\
\varepsilon_{e \mu}^{*} & \varepsilon_{\mu \mu} & \varepsilon_{\mu \tau} \\
\varepsilon_{e \tau}^{*} & \varepsilon_{\mu \tau}^{*} & \varepsilon_{\tau \tau}
\end{array}\right)
$$

where the standard interactions are accounted by the " $1+$ " term in the ee entry, and the non-standard interactions are accounted by the $\varepsilon_{\alpha \beta}$ terms. Since $H_{\text {mat }}$ is Hermitian and its trace is irrelevant for oscillations, we have 8 parameters.

In Ref. [54] an alternative parametrization for $H_{\text {mat }}$, mimicking the structure of the vacuum term in Eq. (1.2), was introduced as

$$
H_{\text {mat }}=Q_{\text {rel }} U_{\text {mat }} D_{\text {mat }} U_{\text {mat }}^{\dagger} Q_{\text {rel }}^{\dagger} \text { with }\left\{\begin{aligned}
Q_{\text {rel }} & =\operatorname{diag}\left(e^{i \alpha_{1}}, e^{i \alpha_{2}}, e^{-i \alpha_{1}-i \alpha_{2}}\right), \\
U_{\text {mat }} & =R_{12}\left(\varphi_{12}\right) \tilde{R}_{13}\left(\varphi_{13}, \delta_{\mathrm{NS}}\right) R_{23}\left(\varphi_{23}\right), \\
D_{\text {mat }} & =\sqrt{2} G_{F} N_{e}(r) \operatorname{diag}\left(\varepsilon, \varepsilon^{\prime}, 0\right)
\end{aligned}\right.
$$


where we denote by $R_{i j}\left(\varphi_{i j}\right)$ a rotation of angle $\varphi_{i j}$ in the $i j$ plane and $\tilde{R}_{13}\left(\varphi_{13}, \delta_{\mathrm{NS}}\right)$ is a complex rotation by angle $\psi_{13}$ and phase $\delta_{\mathrm{NS}}$. Just as Eq. (2.7) this parametrization also contains 8 real parameters: 2 eigenvalues, 3 angles and 3 phases. The two phases $\alpha_{1}$ and $\alpha_{2}$ included in $Q_{\text {rel }}$ are not a feature of neutrino-matter interactions, but rather a relative feature of the vacuum and matter term: they would become unphysical if any of the two terms were not there. Reinterpreted in the notation of Eq. (2.6), this means that only one particular combination of the three complex phases of $\varepsilon_{e \mu}, \varepsilon_{e \tau}, \varepsilon_{\mu \tau}$ is a genuine property of NSI. In other words, the relation in Eq. (2.5) implies that the matter potential behaves as composed of a unique effective fermion, and in this case, as discussed in the previous section, it is a matter of convention to define the off-diagonal elements of the matter potential as three complex parameters, or as three positive real parameters plus a matter CP phase, and the two additional phases being assigned to either vacuum or matter part.

Further simplification follows from neglecting $\Delta m_{21}^{2}$ in the analysis of atmospheric, LBL and all reactor experiments but KamLAND, and by imposing that two eigenvalues of the $H_{\text {mat }}$ are equal $\left(\varepsilon^{\prime}=0\right)$. In the limit $\Delta m_{21}^{2} \rightarrow 0$ the $\theta_{12}$ angle and the $\delta_{\mathrm{CP}}$ phase become unphysical, even in the presence of the generalized $H_{\text {mat }}$ in Eq. (2.7). Similarly, for $\varepsilon^{\prime} \rightarrow 0$ the $\varphi_{23}$ angle and the $\delta_{\mathrm{NS}}$ phase become unphysical and the general $H_{\text {mat }}$ contains 5 real parameters: $\varepsilon$ which represents a rescaling of the matter potential strength, $\varphi_{12}$ and $\varphi_{13}$ which allows for projection of the potential into the $\nu_{\mu}$ and $\nu_{\tau}$ flavors, and the 2 vacuummatter relative phases $\alpha_{1}$ and $\alpha_{2}$. In Ref. [38] it was shown that strong cancellations in the oscillation of atmospheric neutrinos occur when two eigenvalues of $H_{\text {mat }}$ are equal, so that although the limit $\varepsilon^{\prime}=0$ considered here is only a subspace of the most general case on non-standard interactions, it is precisely in this subspace where the weakest constraints can be placed. Under these assumptions the relations between the original $\varepsilon_{\alpha \beta}$ in Eq. (2.6) and the parameters in Eq. (2.7) read:

$$
\begin{aligned}
\varepsilon_{e e}-\varepsilon_{\mu \mu} & =\varepsilon\left(\cos ^{2} \varphi_{12}-\sin ^{2} \varphi_{12}\right) \cos ^{2} \varphi_{13}-1, \\
\varepsilon_{\tau \tau}-\varepsilon_{\mu \mu} & =\varepsilon\left(\sin ^{2} \varphi_{13}-\sin ^{2} \varphi_{12} \cos ^{2} \varphi_{13}\right), \\
\varepsilon_{e \mu} & =-\varepsilon \cos \varphi_{12} \sin \varphi_{12} \cos ^{2} \varphi_{13} e^{i\left(\alpha_{1}-\alpha_{2}\right)}, \\
\varepsilon_{e \tau} & =-\varepsilon \cos \varphi_{12} \cos \varphi_{13} \sin \varphi_{13} e^{i\left(2 \alpha_{1}+\alpha_{2}\right)}, \\
\varepsilon_{\mu \tau} & =\varepsilon \sin \varphi_{12} \cos \varphi_{13} \sin \varphi_{13} e^{i\left(\alpha_{1}+2 \alpha_{2}\right)},
\end{aligned}
$$

which makes explicit that the diagonal terms $\left(\varepsilon_{e e}, \varepsilon_{\mu \mu}, \varepsilon_{\tau \tau}\right)$ can only be determined up to an overall additive constant. The term " -1 " at the end of $\varepsilon_{e e}-\varepsilon_{\mu \mu}$ arises from the standard matter term. The fermion-specific coefficients $\varepsilon_{\alpha \beta}^{f}$ are obtained from the effective ones $\varepsilon_{\alpha \beta}$ just by rescaling:

$$
\varepsilon_{\alpha \beta}^{e}=\varepsilon_{\alpha \beta}, \quad \varepsilon_{\alpha \beta}^{u}=\varepsilon_{\alpha \beta} / Y_{u}, \quad \varepsilon_{\alpha \beta}^{d}=\varepsilon_{\alpha \beta} / Y_{d} .
$$

Thus, in summary, the relevant flavor transition probabilities for atmospheric and LBL experiments depend on eight parameters: $\left(\Delta m_{31}^{2}, \theta_{13}, \theta_{23}\right)$ for the vacuum part, $\left(\varepsilon, \varphi_{12}\right.$, $\left.\varphi_{13}\right)$ for the matter part, and $\left(\alpha_{1}, \alpha_{2}\right)$ as relative phases. As for reactor experiments other 
than KamLAND, matter effects are completely irrelevant due to the very small amount of matter crossed, so the corresponding $P_{e e}$ survival probability only depends on the two parameters $\left(\Delta m_{31}^{2}, \theta_{13}\right)$.

As shown in Appendix B of Ref. [54], only the relative sign of $\Delta m_{31}^{2}$ and $\varepsilon$ is relevant for atmospheric and LBL neutrino oscillations. Concerning the angles, in the general case of unconstrained $\alpha_{i}$ it is enough to consider $0<\theta_{i j}<\pi / 2$ and $0<\varphi_{i j}<\pi / 2$, whereas for the case of real NSI (corresponding to $\alpha_{i} \in\{0, \pi\}$ ) we can set $\alpha_{1}=\alpha_{2}=0$ and extend the $\varphi_{i j}$ range to $-\pi / 2<\varphi_{i j}<\pi / 2$.

\subsection{Earth matter potential for solar and KamLAND neutrinos}

For the study of propagation of solar and KamLAND neutrinos one can work in the one mass dominance approximation, $\Delta m_{31}^{2} \rightarrow \infty$ (which effectively means that generically $\left.G_{F} \sum_{f} N_{f}(r) \varepsilon_{\alpha \beta}^{f} \ll \Delta m_{31}^{2} / E_{\nu}\right)$. In this approximation the survival probability $P_{e e}$ can be written as $[20,56]$

$$
P_{e e}=c_{13}^{4} P_{\text {eff }}+s_{13}^{4}
$$

where $c_{i j} \equiv \cos \theta_{i j}$ and $s_{i j} \equiv \sin \theta_{i j}$. The probability $P_{\text {eff }}$ can be calculated in an effective $2 \times 2$ model with the Hamiltonian $H_{\text {eff }}=H_{\text {vac }}^{\text {eff }}+H_{\text {mat }}^{\text {eff }}$, where:

$$
\begin{aligned}
H_{\mathrm{vac}}^{\mathrm{eff}} & =\frac{\Delta m_{21}^{2}}{4 E_{\nu}}\left(\begin{array}{rr}
-\cos 2 \theta_{12} & \sin 2 \theta_{12} \\
\sin 2 \theta_{12} & \cos 2 \theta_{12}
\end{array}\right), \\
H_{\mathrm{mat}}^{\mathrm{eff}} & =\sqrt{2} G_{F} N_{e}(r)\left(\begin{array}{cc}
c_{13}^{2} & 0 \\
0 & 0
\end{array}\right)+\sqrt{2} G_{F} \sum_{f} N_{f}(r)\left(\begin{array}{rr}
-\varepsilon_{D}^{f} & \varepsilon_{N}^{f} \\
\varepsilon_{N}^{f *} & \varepsilon_{D}^{f}
\end{array}\right) .
\end{aligned}
$$

The coefficients $\varepsilon_{D}^{f}$ and $\varepsilon_{N}^{f}$ are related to the original parameters $\varepsilon_{\alpha \beta}^{f}$ by the following relations:

$$
\begin{aligned}
\varepsilon_{D}^{f}= & c_{13} s_{13} \operatorname{Re}\left[e^{i \delta_{\mathrm{CP}}}\left(s_{23} \varepsilon_{e \mu}^{f}+c_{23} \varepsilon_{e \tau}^{f}\right)\right]-\left(1+s_{13}^{2}\right) c_{23} s_{23} \operatorname{Re}\left(\varepsilon_{\mu \tau}^{f}\right) \\
& -\frac{c_{13}^{2}}{2}\left(\varepsilon_{e e}^{f}-\varepsilon_{\mu \mu}^{f}\right)+\frac{s_{23}^{2}-s_{13}^{2} c_{23}^{2}}{2}\left(\varepsilon_{\tau \tau}^{f}-\varepsilon_{\mu \mu}^{f}\right), \\
\varepsilon_{N}^{f}= & c_{13}\left(c_{23} \varepsilon_{e \mu}^{f}-s_{23} \varepsilon_{e \tau}^{f}\right)+s_{13} e^{-i \delta_{\mathrm{CP}}}\left[s_{23}^{2} \varepsilon_{\mu \tau}^{f}-c_{23}^{2} \varepsilon_{\mu \tau}^{f *}+c_{23} s_{23}\left(\varepsilon_{\tau \tau}^{f}-\varepsilon_{\mu \mu}^{f}\right)\right],
\end{aligned}
$$

so effectively the relevant probabilities for solar and KamLAND neutrinos depend on the 3 real oscillation parameters $\Delta m_{21}^{2}, \theta_{12}$, and $\theta_{13}$ as well as one real $\varepsilon_{D}^{f}$ and one complex $\varepsilon_{N}^{f}$ matter parameter for each $f$. Notice also that the matter chemical composition of the Sun varies substantially along the neutrino production region, with $Y_{n}$ dropping from about $1 / 2$ in the center to about $1 / 6$ at the border of the solar core. Therefore, unlike the case of Eq. (2.5) for the Earth it is not possible to introduce a common set of parameters accounting simultaneously for all the different $f$. Consequently in the analysis of solar data we will consider only one particular choice of $f=e, f=u$ or $f=d$ at a time.

Concerning the parameter ranges, the situation is very similar to the standard case without NSI. The angle $\theta_{13}$ only enters through Eq. (2.10), so it is sufficient to consider $0 \leq \theta_{13} \leq \pi / 2$. The Hamiltonian (2.12) is invariant under the transformation $\Delta m_{21}^{2} \rightarrow$ 
$-\Delta m_{21}^{2} \wedge \theta_{12} \rightarrow \theta_{12}+\pi / 2$, so without loss of generality we can assume $\Delta m_{21}^{2}>0$. In Eq. (2.11) $\theta_{12}$ appears multiplied by 2 , so we can restrict its range to $-\pi / 2 \leq \theta_{12} \leq+\pi / 2$. Finally, the probabilities are insensitive to the overall sign of the non-diagonal entry of (2.12), resulting in a symmetry $\theta_{12} \rightarrow-\theta_{12} \wedge \varepsilon_{N}^{f} \rightarrow-\varepsilon_{N}^{f}$, which can be used to further restrict the $\theta_{12}$ range to $0 \leq \theta_{12} \leq \pi / 2$. Thus in the most general case we have $\Delta m_{21}^{2}>0$, $0 \leq \theta_{i j} \leq \pi / 2, \varepsilon_{D}^{f}$ real, and $\varepsilon_{N}^{f}$ complex. Notice however that, as discussed before, from the point of view of neutrino oscillations the phase of $\varepsilon_{N}^{f}$ is not a genuine NSI property but rather a relative feature of the vacuum and matter parts.

In the specific case of non-standard interactions with electrons $(f=e)$ there is another exact symmetry. Both the standard and the non-standard terms in Eq. (2.12) scale with the same matter density profile $N_{e}(r)$, so they can be merged into a single term and $H_{\text {mat }}^{\text {eff }}$ takes the form:

$$
H_{\mathrm{mat}}^{\mathrm{eff}}=\sqrt{2} G_{F} N_{e}(r)\left(\begin{array}{cc}
-\varepsilon_{D}^{e}+c_{13}^{2} / 2 & \varepsilon_{N}^{e} \\
\varepsilon_{N}^{e *} & \varepsilon_{D}^{e}-c_{13}^{2} / 2
\end{array}\right) .
$$

The probabilities are invariant under $H \rightarrow-H^{*}$, which is realized for $\Delta m_{21}^{2} \rightarrow-\Delta m_{21}^{2} \wedge$ $\left(\varepsilon_{D}^{e}-c_{13}^{2} / 2\right) \rightarrow-\left(\varepsilon_{D}^{e}-c_{13}^{2} / 2\right) \wedge \varepsilon_{N}^{e} \rightarrow-\varepsilon_{N}^{e *}$. Combining this with the general symmetries discussed above we can reabsorb the sign flip of both $\Delta m_{21}^{2}$ and $\varepsilon_{N}^{e}$ into $\theta_{12}$, resulting in the transformation $\theta_{12} \rightarrow \pi / 2-\theta_{12} \wedge \varepsilon_{D}^{e} \rightarrow c_{13}^{2}-\varepsilon_{D}^{e} \wedge \varepsilon_{N}^{e} \rightarrow \varepsilon_{N}^{e *}$. This invariance implies that for each point in the so-called "light-side" of the parameter space (i.e., the region with $\theta_{12}<45^{\circ}$ ) there is a point in the "dark-side" (the region with $\theta_{12}>45^{\circ}$ ) which cannot be distinguished experimentally by oscillations alone. In the case of NSI with $f=u$ or $f=d$ such a symmetry is no longer exact, however as we will see in Sec. 3 it is still realized with considerable accuracy.

As mentioned in Sec. 2.1 the transition probabilities in the atmospheric+LBL sector are invariant under a simultaneous sign flip of $\Delta m_{31}^{2}, \varepsilon$ and $\alpha_{i}$. If this transformation is extended to the solar+KamLAND sector through Eqs. (2.8), (2.9) and then (2.13), (2.14) (with $\delta_{\mathrm{CP}}=0$ as in the atmospheric approximation) it leads to $\varepsilon_{D}^{f} \rightarrow c_{13}^{2} / Y_{f}-\varepsilon_{D}^{f} \wedge$ $\varepsilon_{N}^{f} \rightarrow-\varepsilon_{N}^{f *}$. For $f=e$ this transformation becomes an exact symmetry if combined with a sign flip of $\Delta m_{21}^{2}$, as we have just seen. However, for $f=u$ or $f=d$ such symmetry is only approximate, so that the inclusion of solar data can (at least in principle) lift the sign degeneracy between $\Delta m_{31}^{2}$ and $\varepsilon$.

\section{Analysis of solar and KamLAND data}

Let us start by presenting the results of the updated analysis of solar and KamLAND experiments in the context of oscillations with the generalized matter potential in Eq. (2.12). For KamLAND we include the observed energy spectrum in the DS-1 and DS-2 data sets [57] with a total exposure of $3.49 \times 10^{32}$ target-proton-year (2135 days). In the analysis of solar neutrino experiments we include the total rates from the radiochemical experiments Chlorine [58], Gallex/GNO [59] and SAGE [60]. For real-time experiments we include the 44 data points of Super-Kamiokande phase I (SK1) energy-zenith spectrum [61], the 33 data points of SK2 [62] and 42 data points of SK3 [63] energy and day/night spectra, and the 24 data points of the 1097-day energy spectrum and day-night asymmetry of SK4 [64]. We 
also include the main set of the 740.7 days of Borexino data [65] as well as their high-energy spectrum from 246 live days [66].

The results of the three phases of SNO are included in two different forms. First, we perform our own combined analysis of the 34 data points of the day-night spectrum data of SNO-I [67], the 38 data points of the day-night spectrum of SNO-II [68] and the three total rates of SNO-III [69]. We label this analysis as SNO-DATA. Second, we use the results of their the low energy threshold analysis of the combined SNO phases I-III [70] which is given in the form of an effective $M S W$-like polynomial parametrization for the day and night survival probabilities - under the assumption of unitarity of the oscillation probabilities - in terms of 7 parameters for which the collaboration give the best fit values and covariant matrix. We label this analysis as SNO-POLY. Strictly the results of this effective parametrization cannot be used for study of exotic scenarios in which either unitarity in the active neutrino sector does not hold (like for scenarios with sterile neutrinos) or the energy dependence of the oscillation probability cannot be well represented by a simple quadratic function. Thus in order to verify the robustness of our conclusions on the matter potential we present our results for both variants of the SNO analysis. In both cases we have used the solar fluxes from the Standard Solar Model GS98 [71, 72].

We present the results of the analysis of solar and KamLAND data in Figs. 1 and 2. The presence of NSI with electrons, $f=e$, would affect not only neutrino propagation in matter as described in Eq. (2.12), but also the neutrino-electron cross-section in experiments such as SK and Borexino. Since here we are only interested in studying the bounds to propagation effects we will consider only the cases $f=u$ and $f=d$. Also for simplicity the results are shown for real $\varepsilon_{N}^{f}$. Strictly speaking, as discussed in Sec. 2.2, the sign of $\varepsilon_{N}^{f}$ is not physically observable in oscillation experiments, as it can be reabsorbed into a redefinition of the sign of $\theta_{12}$. However, for definiteness we have chosen to present our results in the convention $\theta_{12} \geq 0$, and therefore we consider both positive and negative values of $\varepsilon_{N}^{f}$.

Fig. 1 shows the two-dimensional projections on the oscillation parameters $\left(\Delta m_{21}^{2}\right.$, $\left.\sin ^{2} \theta_{12}\right)$ and the matter potential parameters $\left(\varepsilon_{N}^{f}, \varepsilon_{D}^{f}\right)$ with $f=u, d$ after marginalizing on the undisplayed parameters, for a fix value of $\sin ^{2} \theta_{13}=0.023$ which is the best fit for the global analysis for $3 \nu$ oscillations $[8,9]$. The first thing to notice is that for both SNO-DATA and SNO-POLY variants there are two disconnected regions in the parameter space. The leftmost region in each panel, whose projection on the oscillation parameters lies in the first octant of $\theta_{12}\left(0 \leq \theta_{12} \leq 45^{\circ}\right)$ and whose projection on the matter potential parameters contains SM case (i.e., the point $\varepsilon_{N}^{f}=\varepsilon_{D}^{f}=0$ ), corresponds to the variation of the "standard" LMA solution in the presence of NSI, so we will refer to it simply as LMA. The rightmost region in each panel, whose projection on the oscillation parameters lies the second octant of $\theta_{12}\left(45^{\circ} \leq \theta_{12} \leq 90^{\circ}\right)$ and whose projection on the matter potential does not contain the SM point $\varepsilon_{N}^{f}=\varepsilon_{D}^{f}=0$, corresponds to the "dark-side" solution found in Ref. [73] where it was labeled as LMA-D. The existence of this new solution, almost degenerate with the usual one, is consequence of the quasi-symmetry of the matter potential discussed below Eq. (2.15). We find that at present the best fit point is in most of the cases 


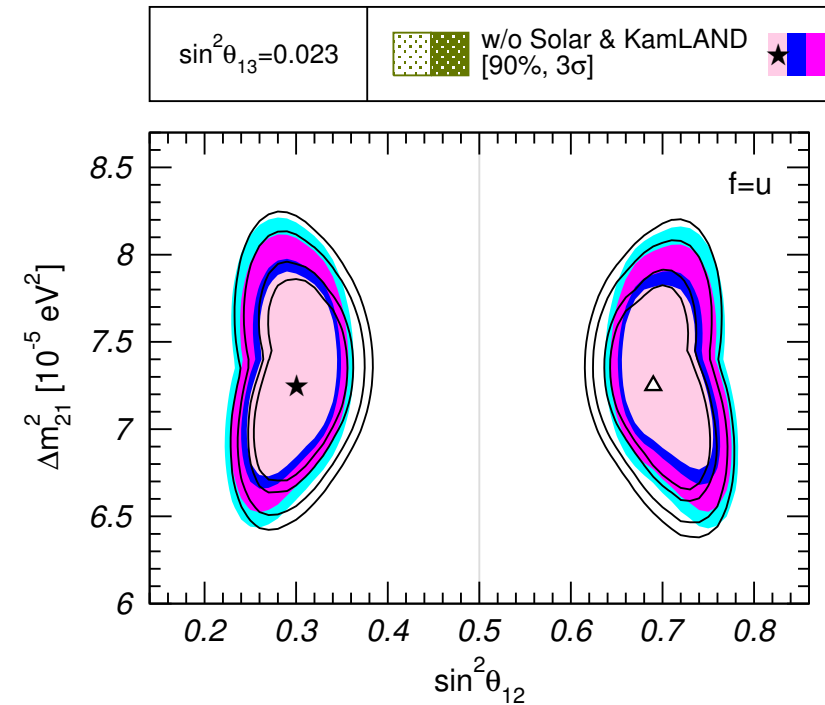

SNO-POLY

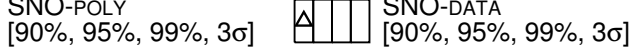
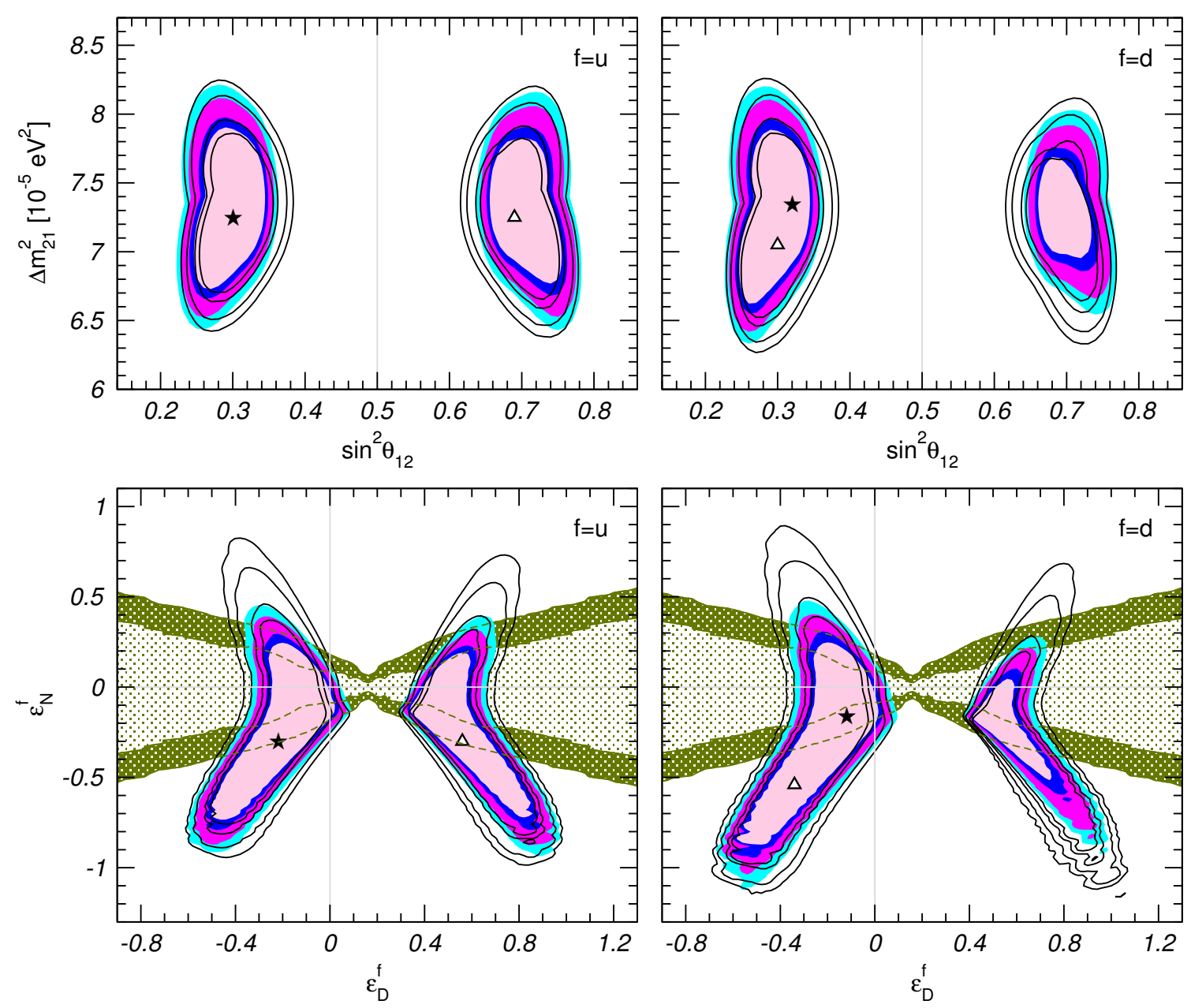

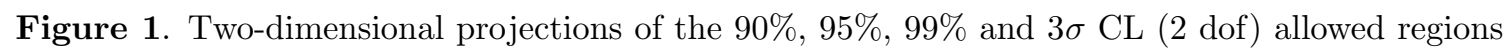
from the analysis of solar and KamLAND data in the presence of non-standard matter potential. The results are shown for a fix value of $\sin ^{2} \theta_{13}=0.023$ and after marginalizing over the two undisplayed parameters. The left (right) panels corresponds to $f=u(f=d)$. The colored filled (black-contour void) regions in each panel correspond to the SNO-POLY (SNO-DATA) variants of the solar analysis, see text for details. The best fit point is marked with a star (triangle). For comparison we show also in the lower panels the two green dotted areas correspond to the $90 \%$ and $3 \sigma$ CL allowed regions from the analysis of the atmospheric and LBL data.

in the LMA region, but LMA-D lies only at a $\Delta \chi^{2}=-0.06(f=u)$ and $0.4(f=d)$ in the SNO-DATA variant, increasing to $\Delta \chi^{2}=0.3(f=u)$ and $1.4(f=d)$ in the SNO-POLY variant. As seen in the lower panels the LMA-D solution requires a non-standard matter potential with quite sizable values of $\varepsilon_{D}^{f}$. An obvious question is whether such large values are in contradiction with other neutrino oscillation data, in particular with atmospheric neutrinos. We will return quantitatively to this point in the next section but for illustration we show also in the lower panels as dotted green regions the corresponding $90 \%$ and $3 \sigma$ 


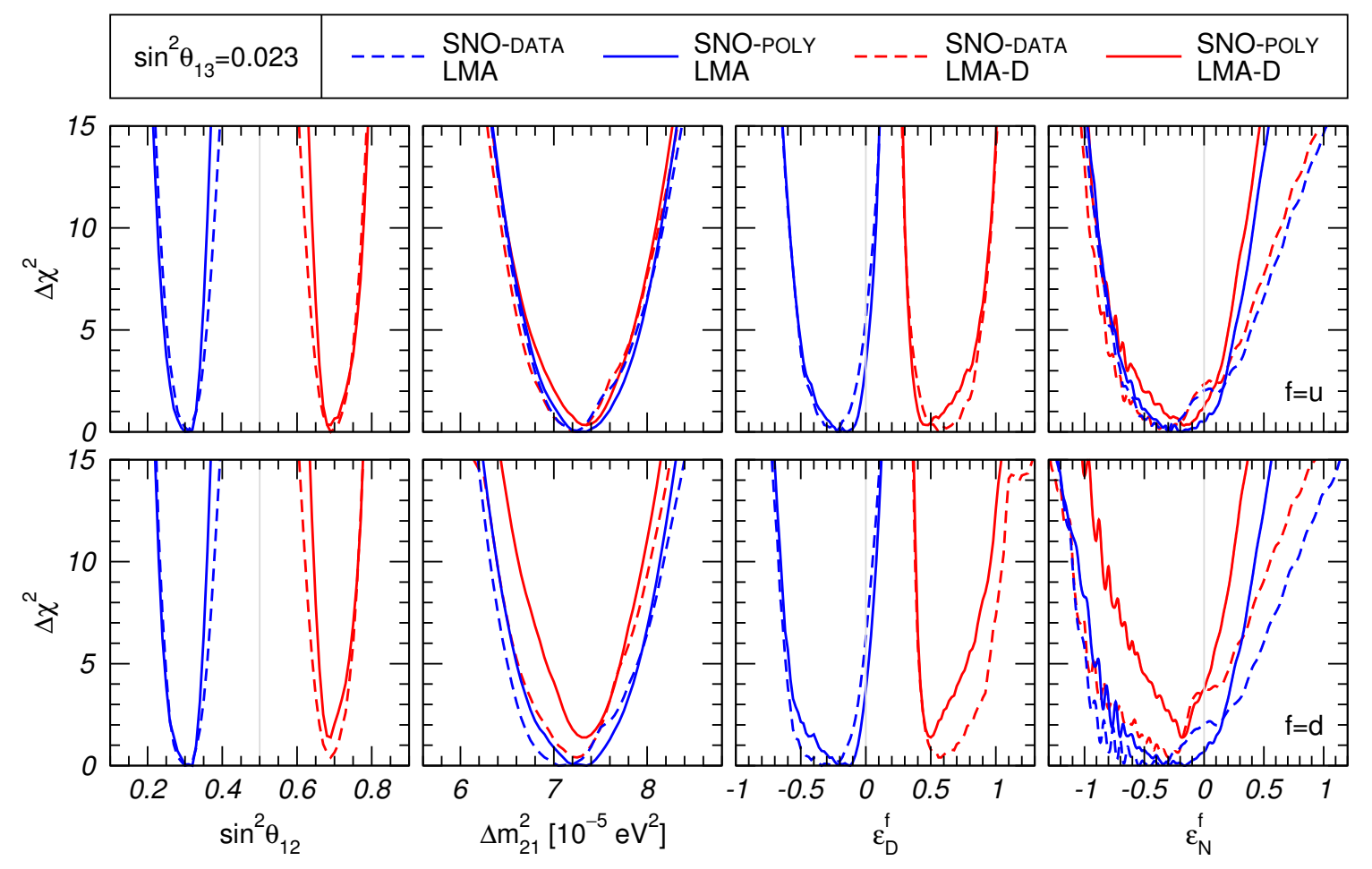

Figure 2. Dependence of the $\Delta \chi^{2}$ function for the analysis of the solar and KamLAND data on the relevant oscillation and matter potential parameters for $f=u$ (upper panels) and $f=d$ (lower panels), for both LMA and LMA-D regions and the two variants of the SNO analysis, as labeled in the figure.

CL (2 dof) from the analysis of atmospheric and LBL experiments. We see from the figure that still a sizable fraction of the required non-standard matter potential parameters for the LMA-D solution is compatible with all the oscillation data.

In what respects the dependence on $f$ the figure shows that although there are small quantitative differences, qualitatively the results are rather similar for non-standard potential for $u$ or $d$ quarks. Also both variants of the SNO analysis yield similar results.

Fig. 2 contains the dependence of $\Delta \chi^{2}$ on each of the four parameters $\Delta m_{21}^{2}, \theta_{12}$, $\varepsilon_{D}^{f}, \varepsilon_{N}^{f}$, again for $\sin ^{2} \theta_{13}=0.023$ after marginalizing over the other three. In each panel the four curves correspond to the LMA and LMA-D solutions for both variants of the SNO analysis. The main feature to notice is that in all cases the fit prefers some nonstandard value of the matter potential parameters, while for any $f$ the SM potential lies at a $\Delta \chi^{2}=5.3$ and $\Delta \chi^{2}=7.9$ for SNO-POLY and SNO-DATA, respectively. This arises from the well-known fact that neither the SNO nor SK4 low energy threshold analysis nor the ${ }^{8} \mathrm{~B}$ measurement in Borexino seem to show evidence of the low energy turn-up of the spectrum predicted in the standard LMA MSW solution. This behavior can be better described in the presence of a non-standard matter potential. This is illustrated in Fig. 3 where we show the survival probability of solar neutrinos as a function of the neutrino energy, for the best fit of oscillations only (black line) as well as the best fits for $f=u$ and $f=d$ in the presence of NSI from the analysis of solar+KamLAND data (red lines) and from the 


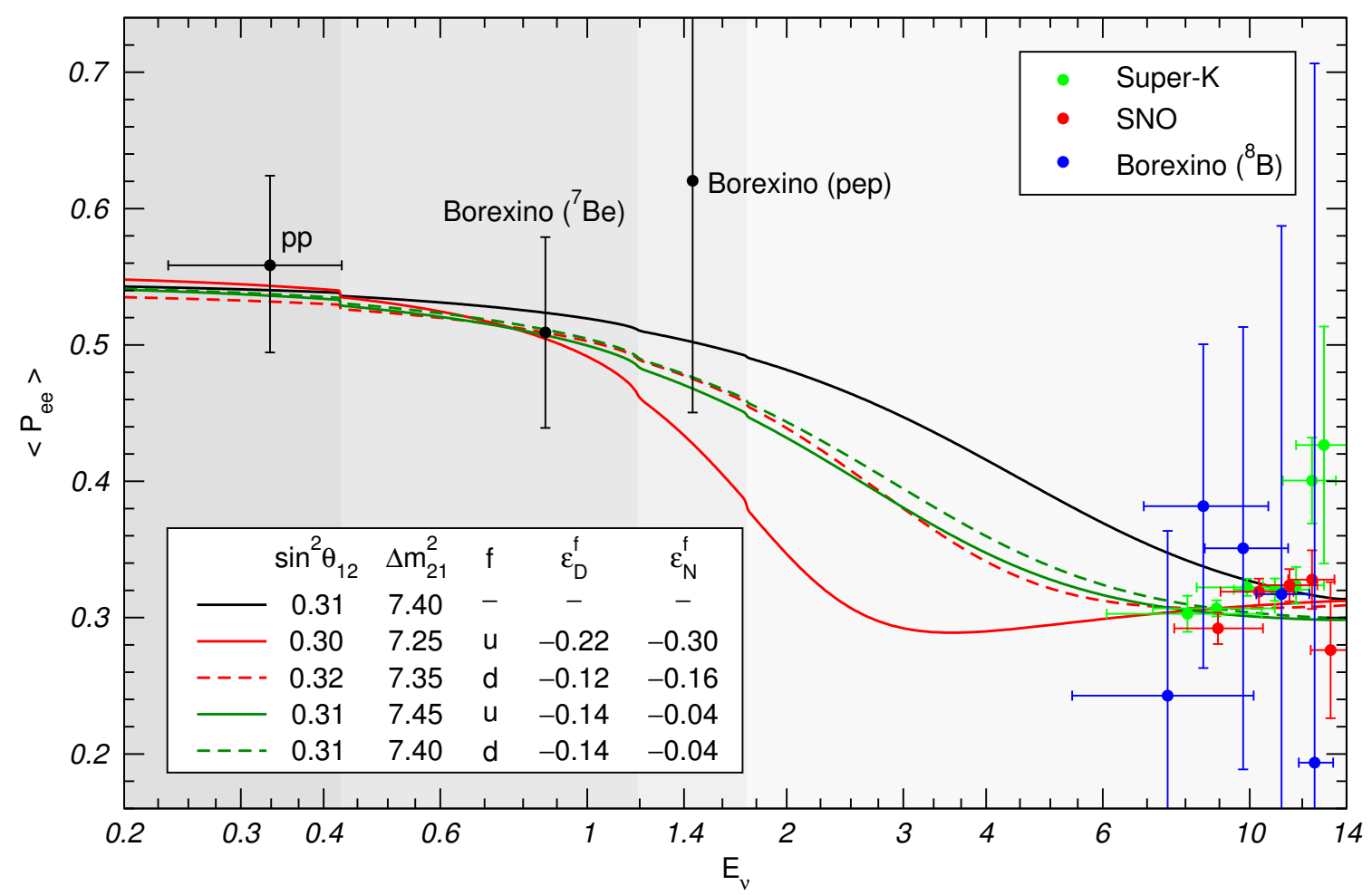

Figure 3. Survival probabilities in the Sun for different sets of oscillation and matter potential parameters as labeled in the figure. In all cases we set $\sin ^{2} \theta_{13}=0.023$; the quoted value of $\Delta m_{21}^{2}$ is given in units of $10^{-5} \mathrm{eV}^{2}$. For illustration we also show the extracted average survival probabilities from different experiments. See text for details.

global analysis discussed in the next section (green lines). In order to take into account the dependence on the neutrino production point, which is of particular relevance in the presence of non-standard matter potential, we define the average survival probability $\left\langle P_{e e}\right\rangle$ as

$$
\left\langle P_{e e}\left(E_{\nu}\right)\right\rangle=\frac{\sum_{i} \Phi_{i}\left(E_{\nu}\right) \int \rho_{i}(r) P_{e e}\left(E_{\nu}, r\right) d r}{\sum_{i} \Phi_{i}\left(E_{\nu}\right)}
$$

where $i=\mathrm{pp}$, pep, ${ }^{7} \mathrm{Be},{ }^{13} \mathrm{~N},{ }^{15} \mathrm{O},{ }^{17} \mathrm{~F},{ }^{8} \mathrm{~B}$ and hep labels the neutrino production reaction and $\rho_{i}(r)$ is the distribution of production points for the reaction $i$ normalized to 1.

\section{Results of global analysis}

We now present the results of the global analysis including also atmospheric, LBL and all other reactor data. The data samples included here are the same as in the NuFIT 1.1 analysis described in Ref. [8]. For atmospheric data we use the Super-Kamiokande results from phases 1-4 [74], adding the 1097 days of phase 4 to their published data from phases 1-3 [75]. For what concerns long-baseline accelerator experiments, we combine the energy distribution obtained by MINOS in both $\nu_{\mu}\left(\bar{\nu}_{\nu}\right)$ disappearance [76] and $\nu_{e}\left(\bar{\nu}_{e}\right)$ appearance with $10.7(3.36) \times 10^{20}$ protons on target [77], and T2K $\nu_{e}$ appearance and $\nu_{\mu}$ disappearance data for phases $1-3$ corresponding to $3.01 \times 10^{20}$ pot [78]. For oscillation signals at 
reactor experiments, besides KamLAND, we include data from the finalized experiments CHOOZ [79] (energy spectrum data) and Palo Verde [80] (total rate) together with the recent spectrum from Double Chooz with 227.9 days live time [81], and the total even rates in the near and far detectors in Daya Bay [82] and Reno with 402 days of data-taking [83]. For the reactor fluxes we follow here the approach of Ref. [84], i.e., we introduce an overall flux normalization which is then fitted to the data together with the oscillation and matter potential parameters. To better constrain such reactor flux normalization we also include in the analysis the results of the reactor experiments Bugey4 [85], ROVNO4 [86], Bugey3 [87], Krasnoyarsk [88, 89], ILL [90], Gösgen [91], SRP [92], and ROVNO88 [93], which due to their short baselines $(L \lesssim 100 \mathrm{~m})$ are insensitive to the neutrino oscillation effects discussed here.

We present the results of the global analysis in Figs. 4, 5, and 6. In Fig. 4 we display the two-dimensional projections of the allowed regions in the matter potential parameters $\varepsilon, \varphi_{12}$ and $\varphi_{13}$ (in the parametrization of Eq. (2.7) with the additional constraint of equal matter eigenvalues $\varepsilon^{\prime}=0$ ) after marginalizing over the oscillation parameters $\Delta m_{21}^{2}, \Delta m_{31}^{2}$, $\theta_{12}, \theta_{23}$, and $\theta_{13}$. Since the $\alpha_{i}$ phases have little impact on our results, we set for simplicity $\alpha_{1}=\alpha_{2}=0$. Also, for the sake of concreteness we focus here on $f=u$. The filled colored (black-contour void) regions correspond to the global analysis with the SNO-POLY (SNODATA) variant of the solar data. For comparison we show also the dotted green regions which correspond to the analysis of atmospheric, LBL and reactor neutrinos (without solar nor KamLAND) and therefore update our previous results of Ref. [54]. As discussed in Refs. $[38,39,54]$ no bound on the magnitude of the matter effects, $\varepsilon$, can be derived from the analysis of atmospheric and LBL experiments in this general scenario. Specific bounds on $\varepsilon$ can be derived if a certain flavor structure of the matter potential is assumed a priori (for example, if we assume that no matter effects are present in the $e \mu$ and $e \tau$ projections, which corresponds to $\varphi_{12}=\pi / 2$ ), implying that $\varphi_{12}$ and/or $\varphi_{13}$ are larger than some given value. Conversely when marginalizing over $\varepsilon$ the full flavor projection $\left(\varphi_{12}, \varphi_{13}\right)$ plane is allowed. However, as seen from the figure, once the results of solar and KamLAND experiments (i.e., the samples involving $\nu_{e}$ or $\bar{\nu}_{e}$ and long enough distances to see both oscillations and NSI effects) are included in the analysis, a bound on the magnitude of the matter effects $\varepsilon$ is obtained. Furthermore the flavor structure of the potential is dramatically constrained as seen in upper-left panel.

Fig. 5 shows the two-dimensional projections of the allowed regions from our global analysis in different combinations of the oscillation parameters, again for $f=u$. The regions are obtained after marginalizing over the undisplayed oscillation and matter potential parameters. For comparison we also show as black-contour void regions the corresponding results with the usual SM matter potential. ${ }^{1}$ The figure clearly shows the robustness of the determination of the oscillation parameters even in the presence of a generalized matter potential, with the exception of the octant of $\theta_{12}$. In this respect, we find that the

\footnotetext{
${ }^{1}$ Notice that in this analysis we are neglecting $\Delta m_{21}^{2}$ effects in the atmospheric and LBL oscillations, hence the standard oscillation results have no sensitivity to $\mathrm{CP}$ violation and only very marginal sensitivity to the mass ordering and the $\theta_{23}$ octant. For fully updated results and a complete treatment of neutrino oscillations in the standard case we address the reader to Refs. [8, 9].
} 


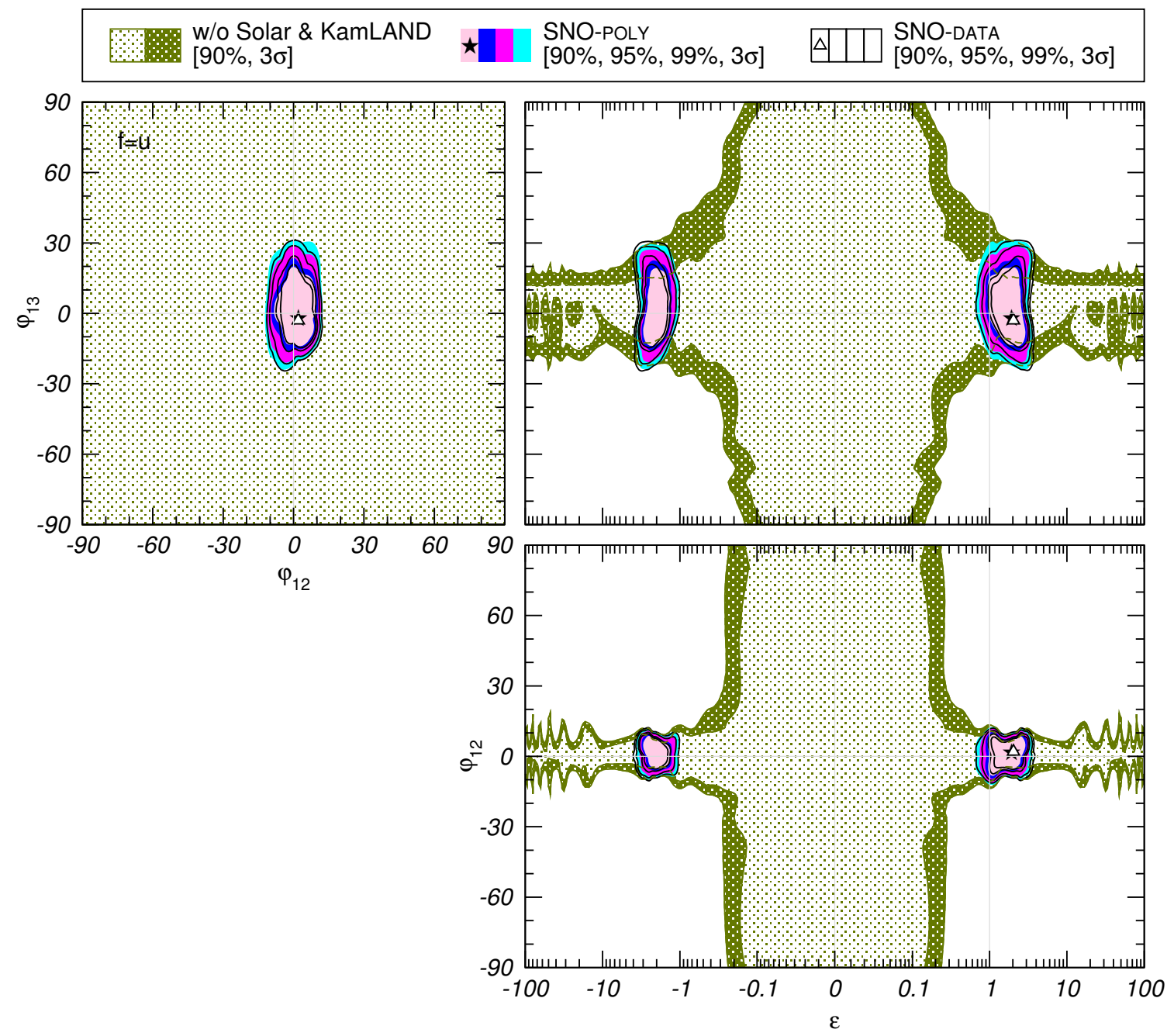

Figure 4. Two-dimensional projections on the matter potential parameters $\left(\varepsilon, \varphi_{12}, \varphi_{13}\right)$ of the $90 \%$, $95 \%, 99 \%$ and $3 \sigma$ CL (2 dof) allowed regions from the global analysis of solar, atmospheric, reactor and LBL data after marginalization with respect to the undisplayed parameters. The colored filled (black-contour void) regions in each panel correspond to $f=u$ and the SNO-POLY (SNO-DATA) variants of the solar analysis. The best fit point is marked with a star (triangle). For comparison we also show as green dotted areas the $90 \%$ and $3 \sigma$ CL regions from the analysis of atmospheric, LBL and reactor neutrinos (without solar nor KamLAND).

LMA-D solution is still allowed in the global analysis at $\Delta \chi^{2}=0.1(0.2)$ for $f=u$ and the SNO-DATA (SNO-POLY) variants, and at $\Delta \chi^{2}=1.1$ (1.9) for $f=d$ and the SNO-DATA (SNO-POLY) analysis. In the figure we also show as green or red dotted regions the results of the analysis when the effects of the non-standard matter parameters are neglected in either Solar+KamLAND (green, upper panels) or in atmospheric+LBL (red, lower panels). The comparison of the global analysis with these partial analyses illustrates the complementarity of the solar+KamLAND and the atmospheric + LBL data in the robustness of the global fit. We also notice how in the upper panels the green regions are perfectly symmetric under a sign flip of $\Delta m_{31}^{2}$, as explained at the end of Sec. 2.1. However, for 

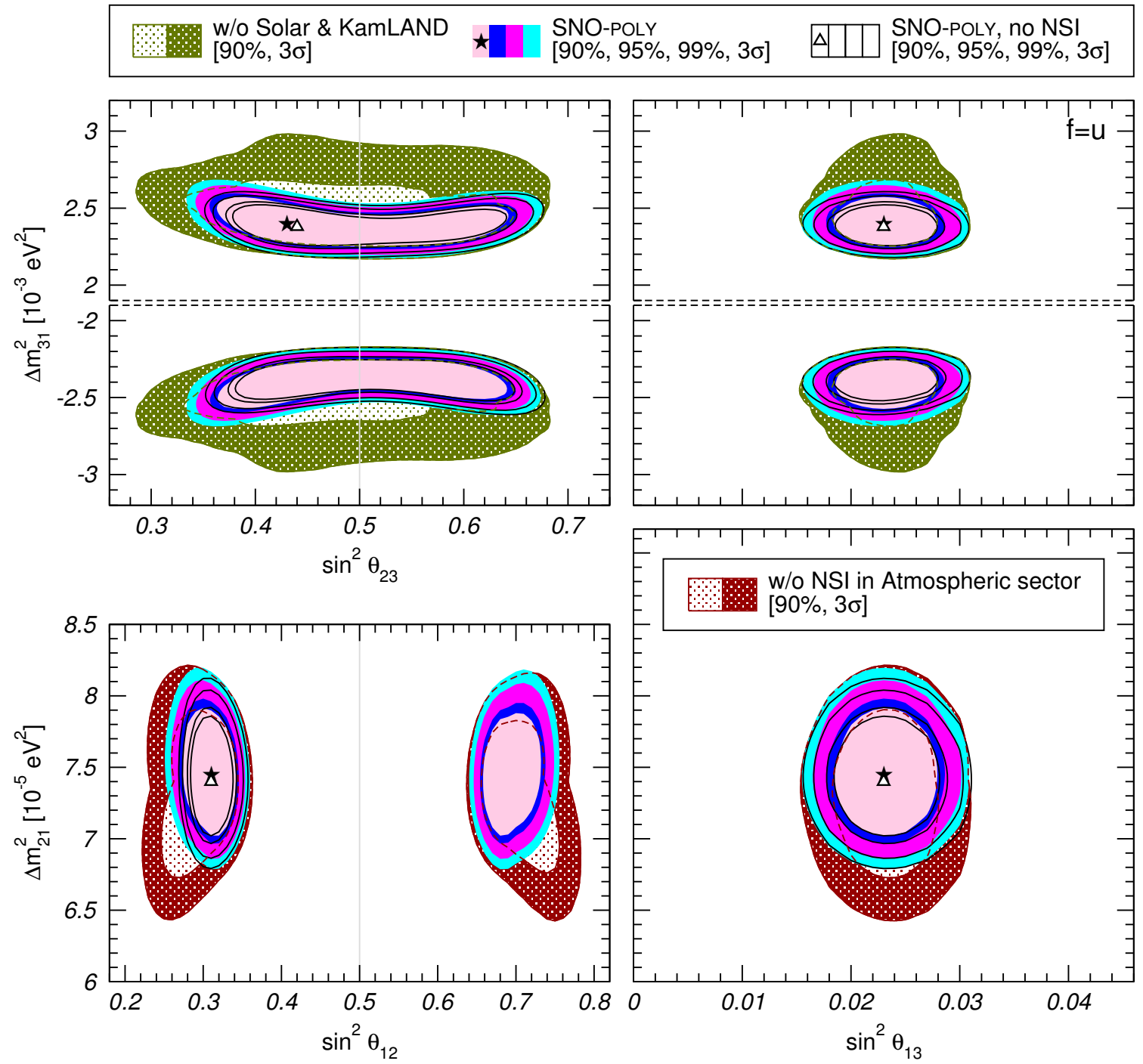

Figure 5. Two-dimensional projections of the 90\%, 95\%, 99\% and $3 \sigma$ CL (2 dof) allowed regions of the oscillation parameters for $f=u$ and the SNO-POLY variant of the solar analysis, after marginalizing over the matter potential parameters and the undisplayed oscillation parameters. The full regions and the star correspond to the global analysis including NSI, while the blackcontour void regions and the triangle correspond to the analysis with the usual SM potential. The green and red dotted areas show the $90 \%$ and $3 \sigma$ CL allowed regions from partial analyses where the effects of the non-standard matter potential have been neglected either in the solar+KamLAND (green) or in the atmospheric+LBL (red) sectors.

NSI with quarks $(f=u, d)$ this degeneracy is lifted once the solar data are also included in the analysis, as discussed in Sec. 2.2. Thus the colored regions are not exactly identical for both orderings, although with present data the asymmetry is still minimal.

In Fig. 6 we plot the dependence of the $\Delta \chi^{2}$ function for the global analysis on the NSI parameters $\varepsilon_{\alpha \beta}^{f}$, after marginalizing over the undisplayed oscillation and matter potential parameters. Similarly, in Fig. 7 we show the present determination on the effective mat- 


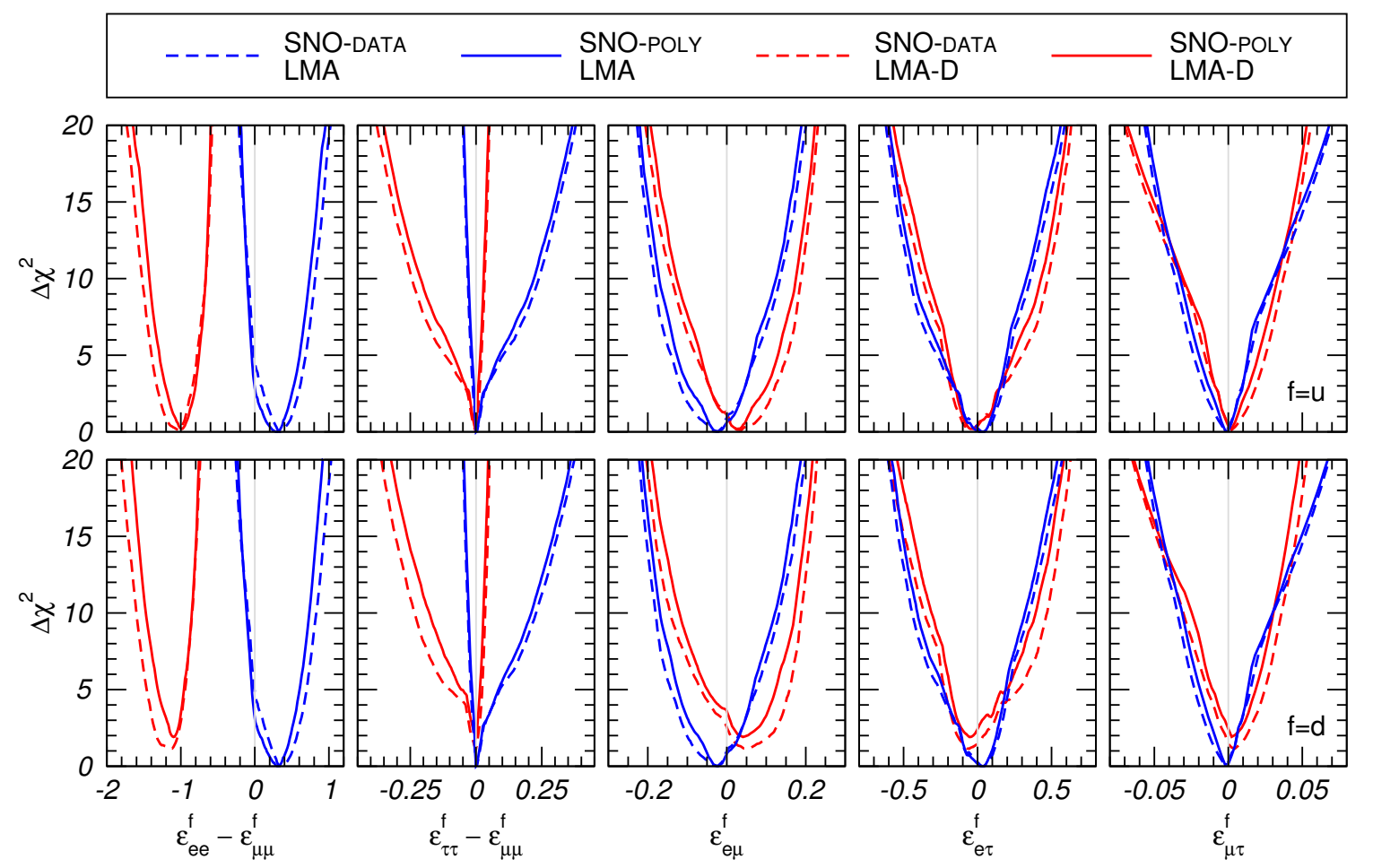

Figure 6. Dependence of the $\Delta \chi^{2}$ function for the global analysis of solar, atmospheric, reactor and LBL data on the NSI parameters $\varepsilon_{\alpha \beta}^{f}$ for $f=u$ (upper panels) and $f=d$ (lower panels), for both LMA and LMA-D regions and the two variants of the SNO analysis, as labeled in the figure.

ter potential parameters $\varepsilon_{D}^{f}$ and $\varepsilon_{N}^{f}$ relevant in the propagation of solar and KamLAND neutrinos. In both figures we display separately the results of the marginalization in the LMA and the LMA-D regions of the parameter space, as well as both the SNO-DATA and SNO-POLY variants of the solar analysis. From these figures we derive the $90 \%$ and $3 \sigma$ allowed ranges for the NSI parameters implied by the global analysis, which we summarize in Table 1. The results in this table correspond to the SNO-POLY analysis and have been obtained for real matter potential parameters. As discussed in Sec. 2, in such a case only the relative sign of the various $\varepsilon_{\alpha \neq \beta}^{f}$ and the vacuum mixing angles can be determined by oscillations. Thus strictly speaking once the results are marginalized with respect to all other parameters in the most general parameter space, the oscillation analysis can only provide bounds on $\left|\varepsilon_{\alpha \neq \beta}^{f}\right|$. Still, for the sake of completeness we have decided to retain in Table 1 the signs of the non-diagonal $\varepsilon_{\alpha \neq \beta}^{f}$, which is correct as long as such signs are understood to be relative vacuum-matter quantities and not intrinsic NSI features.

Neutrino scattering experiments such as CHARM [94, 95], CDHSW [96] and NuTeV [97] are sensitive to NSI with $u$ and $d$, and can therefore yield information on $\varepsilon_{\alpha \beta}^{f}$ [98]. In Ref. [73] it was found that the combination with CHARM scattering results [94, 95] for $f=d$ substantially lifts the statistical difference between LMA and LMA-D. Although a rigorous combined analysis of the oscillation results presented here with those from scatter- 

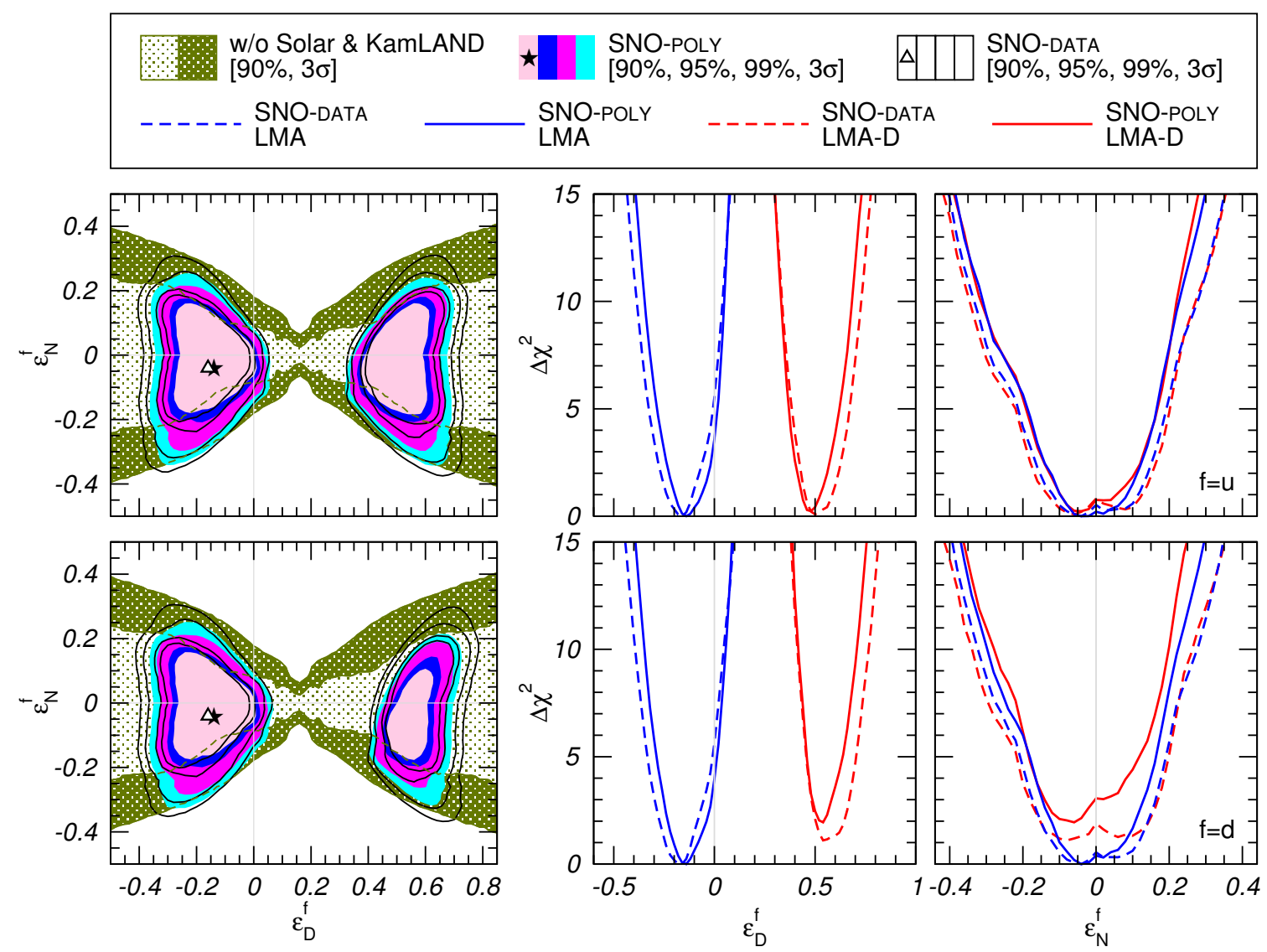

Figure 7. Constraints on the effective matter potential parameters $\varepsilon_{D}^{f}$ and $\varepsilon_{N}^{f}$ relevant in solar neutrino propagation for $f=u$ (upper panels) and $f=d$ (lower panels). In the left panels we show as colored filled (black-contour void) areas the two-dimensional projections of the $90 \%, 95 \%$, $99 \%$ and $3 \sigma$ CL (2 dof) allowed regions from the global analysis, for the SNO-POLY (SNO-DATA) variants of the solar analysis. The best fit point is marked with a star (triangle). The green dotted areas correspond to the $90 \%$ and $3 \sigma$ CL allowed regions from the analysis of atmospheric, LBL and reactor data (without solar and KamLAND). The central and right panels show the dependence of $\Delta \chi^{2}$ from the global analysis on $\varepsilon_{D}^{f}$ and $\varepsilon_{N}^{f}$, as labeled in the figure.

ing experiments is beyond the scope of this paper, ${ }^{2}$ in Table 1 we present separate ranges for marginalization over $0 \leq \theta_{12} \leq 45^{\circ}$ (denoted "LMA") and over the complete parameter space $0 \leq \theta_{12} \leq 90^{\circ}$ (denoted "LMA $\oplus$ LMA-D"), so to give at least an idea of what could be gained from scattering experiments. In most of the cases the LMA $\oplus$ LMA-D marginalization yield just a slightly wider interval than the marginalization within the LMA region. However, for $\varepsilon_{e e}^{f}-\varepsilon_{\mu \mu}^{f}$ and $\varepsilon_{D}^{f}$ the general allowed range is composed by two separated intervals, one arising from the LMA region and the other from the LMA-D region, so the full LMA $\oplus$ LMA-D range has to be intended as the direct sum of the bound provided in the LMA case and the extra interval quoted in the LMA $\oplus$ LMA-D column.

\footnotetext{
${ }^{2}$ Notice that neutrino scattering results also depend on the axial NSI interactions and a rigorous global study of neutrino oscillation and scattering data will contain a larger number of parameters which makes it technically challenging.
} 


\begin{tabular}{|l|c|r|r|rr|}
\hline \multicolumn{2}{|c|}{} & \multicolumn{2}{|c|}{$90 \%$ CL } & \multicolumn{2}{|c|}{$3 \sigma$} \\
\hline Param. & best-fit & LMA & LMA $\oplus$ LMA-D & LMA & LMA $\oplus$ LMA-D \\
\hline \hline$\varepsilon_{e e}^{u}-\varepsilon_{\mu \mu}^{u}$ & +0.298 & {$[+0.00,+0.51]$} & $\oplus[-1.19,-0.81]$ & {$[-0.09,+0.71]$} & $\oplus[-1.40,-0.68]$ \\
$\varepsilon_{\tau \tau}^{u}-\varepsilon_{\mu \mu}^{u}$ & +0.001 & {$[-0.01,+0.03]$} & {$[-0.03,+0.03]$} & {$[-0.03,+0.20]$} & {$[-0.19,+0.20]$} \\
$\varepsilon_{e \mu}^{u}$ & -0.021 & {$[-0.09,+0.04]$} & {$[-0.09,+0.10]$} & {$[-0.16,+0.11]$} & {$[-0.16,+0.17]$} \\
$\varepsilon_{e \tau}^{u}$ & +0.021 & {$[-0.14,+0.14]$} & {$[-0.15,+0.14]$} & {$[-0.40,+0.30]$} & {$[-0.40,+0.40]$} \\
$\varepsilon_{\mu \tau}^{u}$ & -0.001 & {$[-0.01,+0.01]$} & {$[-0.01,+0.01]$} & {$[-0.03,+0.03]$} & {$[-0.03,+0.03]$} \\
\hline$\varepsilon_{D}^{u}$ & -0.140 & {$[-0.24,-0.01]$} & $\oplus[+0.40,+0.58]$ & {$[-0.34,+0.04]$} & $\oplus[+0.34,+0.67]$ \\
$\varepsilon_{N}^{u}$ & -0.030 & {$[-0.14,+0.13]$} & {$[-0.15,+0.13]$} & {$[-0.29,+0.21]$} & {$[-0.29,+0.21]$} \\
\hline \hline$\varepsilon_{e e}^{d}-\varepsilon_{\mu \mu}^{d}$ & +0.310 & {$[+0.02,+0.51]$} & $\oplus[-1.17,-1.03]$ & {$[-0.10,+0.71]$} & $\oplus[-1.44,-0.87]$ \\
$\varepsilon_{\tau \tau}^{d}-\varepsilon_{\mu \mu}^{d}$ & +0.001 & {$[-0.01,+0.03]$} & {$[-0.01,+0.03]$} & {$[-0.03,+0.19]$} & {$[-0.16,+0.19]$} \\
$\varepsilon_{e \mu}^{d}$ & -0.023 & {$[-0.09,+0.04]$} & {$[-0.09,+0.08]$} & {$[-0.16,+0.11]$} & {$[-0.16,+0.17]$} \\
$\varepsilon_{e \tau}^{d}$ & +0.023 & {$[-0.13,+0.14]$} & {$[-0.13,+0.14]$} & {$[-0.38,+0.29]$} & {$[-0.38,+0.35]$} \\
$\varepsilon_{\mu \tau}^{d}$ & -0.001 & {$[-0.01,+0.01]$} & {$[-0.01,+0.01]$} & {$[-0.03,+0.03]$} & {$[-0.03,+0.03]$} \\
\hline$\varepsilon_{D}^{d}$ & -0.145 & {$[-0.25,-0.02]$} & $\oplus[+0.49,+0.57]$ & {$[-0.34,+0.05]$} & $\oplus[+0.42,+0.70]$ \\
$\varepsilon_{N}^{d}$ & -0.036 & {$[-0.14,+0.12]$} & {$[-0.14,+0.12]$} & {$[-0.28,+0.21]$} & {$[-0.28,+0.21]$} \\
\hline
\end{tabular}

Table 1. $90 \%$ and $3 \sigma$ allowed ranges for the matter potential parameters $\varepsilon_{\alpha \beta}^{f}$ for $f=u, d$ as obtained from the global analysis of oscillation data. The results are obtained after marginalizing over oscillation and the other matter potential parameters either within the LMA only and within either LMA or LMA-D subspaces respectively. The numbers quoted are the SNO-POLY variant of the solar analysis. See text for details.

\section{Summary}

In this article we have quantified our current knowledge of the size and flavor structure of the matter background effects in the evolution of solar, atmospheric, reactor and LBL neutrinos based solely on a global analysis of oscillation data. It complements the study in Ref. [54] where the analysis of the matter potential was perform only considering atmospheric and LBL neutrinos.

After briefly presenting the most general parametrization of the matter potential and its connection with non-standard neutrino interactions (NSI), we have focused on the analysis of solar and KamLAND data. We have found (see Fig. 2) that the fit always prefers some non-standard value of the matter potential parameters, while the SM potential lies at a $\Delta \chi^{2} \sim 5-8$ depending on the details of the analysis. This is consequence of the fact that none of the experiments sensitive to ${ }^{8} \mathrm{~B}$ neutrinos has provided so far evidence of the low energy turn-up of the spectrum predicted in the standard LMA MSW solution (see Fig. 3). We have also found in that the present analysis still allows for two disconnected regions in the parameter space, the "standard" LMA region and the "dark side" LMA-D (see Fig. 1), and that the statistical difference between both solutions never exceeds $\Delta \chi^{2}=1.4$. Although the LMA-D solution requires rather large values of the matter parameters, we have shown (and latter quantified in Sec. 4) that it is still fully compatible with the bounds from atmospheric and LBL oscillation data. 
We have then turned to a global analysis in which the data from solar and KamLAND have been combined with those from atmospheric, LBL, and other reactor experiments. For what concerns the impact of the non-standard matter potential on the determination of the oscillation parameters, we found that the determination of $\Delta m_{21}^{2},\left|\Delta m_{31}^{2}\right|, \sin ^{2} \theta_{23}$, and $\sin ^{2} \theta_{13}$ is very robust due to strong synergies between solar+KamLAND and atmospheric+LBL data. In particular, once the results of solar and KamLAND experiments are included in the analysis both the magnitude and the flavor structure of NSI are strongly constrained, thus preventing the weakening of the $\left|\Delta m_{31}^{2}\right|$ and $\theta_{23}$ bounds which was observed in Refs. $[38,39,54]$ from the analysis of atmospheric and LBL data alone. In turn, the inclusion of atmospheric + LBL data in the solar analysis severely constrain the allowed range of non-diagonal NSI described by the effective parameter $\varepsilon_{N}^{f}$, resulting in the stabilization of the $\Delta m_{21}^{2}$ and $\sin ^{2}\left(2 \theta_{12}\right)$ bounds. However, unlike for the case of oscillations with the usual SM potential, in the presence of non-standard interactions a new solution with $\sin ^{2} \theta_{12}>0.5$ (LMA-D region) becomes allowed. With all this, the $3 \sigma$ ranges of the oscillation parameters read:

$$
\begin{aligned}
& \text { Standard Matter Potential } \\
& \text { Generalized Matter Potential } \\
& \sin ^{2} \theta_{12} \in[0.27,0.35], \\
& \sin ^{2} \theta_{23} \in[0.36,0.67], \\
& \sin ^{2} \theta_{13} \in[0.016,0.030], \\
& \Delta m_{21}^{2} \in[6.87,8.03] \times 10^{-5} \mathrm{eV}^{2}, \\
& \left|\Delta m_{31}^{2}\right| \in[2.20,2.58] \times 10^{-3} \mathrm{eV}^{2}, \\
& \sin ^{2} \theta_{12} \in[0.26,0.35] \oplus[0.65,0.75], \\
& \sin ^{2} \theta_{23} \in[0.34,0.67] \text {, } \\
& \sin ^{2} \theta_{13} \in[0.016,0.030] \text {, } \\
& \Delta m_{21}^{2} \in[6.86,8.10] \times 10^{-5} \mathrm{eV}^{2}, \\
& \left|\Delta m_{31}^{2}\right| \in[2.20,2.65] \times 10^{-3} \mathrm{eV}^{2} .
\end{aligned}
$$

The corresponding bounds on the individual NSI parameters from the global analysis after marginalization from all other oscillation and matter parameters are given in Fig. 6 and Table 1. Comparing the results in the Table with the bounds derived in Refs. [98, 99] from non-oscillation data we find that, with the possible exception of $\varepsilon_{e \mu}^{u, d}$, the global oscillation analysis presented here yields the most restrictive bounds on the vector NSI parameters. This is even more impressive if one considers that the one-dimensional bounds in Table 1 arise as projections of a global scan of the entire parameter space, and therefore correlations among different parameters are properly take into account. Conversely, the bounds from neutrino scattering experiments are usually obtained on a one-by-one basis, i.e. varying a single parameter at a time while keeping all the others set to zero. In spite of this, neutrino scattering experiments still provide complementary information to oscillation experiments, for example for $f=d$ they can substantially lifts the degeneracy between the LMA and LMA-D solutions. Therefore, although a rigorous combined analysis of neutrino oscillations and neutrino scattering experiments is technically challenging and well beyond the scope of the present work, it is certainly worth considering for the future.

\section{Acknowledgments}

This work is supported by Spanish MINECO (grants FPA-2010-20807, FPA-2012-31880, FPA-2012-34694, consolider-ingenio 2010 grant CSD-2008-0037 and "Centro de Excelen- 
cia Severo Ochoa" program SEV-2012-0249), by CUR Generalitat de Catalunya (grant 2009SGR502), by Comunidad Autonoma de Madrid (HEPHACOS project S2009/ESP1473), by USA-NSF (grant PHY-09-6739) and by the European Union (EURONU project FP7-212372 and FP7 Marie Curie-ITN actions PITN-GA-2009-237920 "UNILHC" and PITN-GA-2011-289442 "INVISIBLES").

\section{References}

[1] B. Pontecorvo, Neutrino experiments and the question of leptonic-charge conservation, Sov. Phys. JETP 26 (1968) 984-988.

[2] V. N. Gribov and B. Pontecorvo, Neutrino astronomy and lepton charge, Phys. Lett. B28 (1969) 493.

[3] M. C. Gonzalez-Garcia and M. Maltoni, Phenomenology with Massive Neutrinos, Phys. Rept. 460 (2008) 1-129, [arXiv: 0704.1800].

[4] Z. Maki, M. Nakagawa, and S. Sakata, Remarks on the unified model of elementary particles, Prog. Theor. Phys. 28 (1962) 870-880.

[5] M. Kobayashi and T. Maskawa, CP Violation in the Renormalizable Theory of Weak Interaction, Prog. Theor. Phys. 49 (1973) 652-657.

[6] S. M. Bilenky, J. Hosek, and S. T. Petcov, On Oscillations of Neutrinos with Dirac and Majorana Masses, Phys. Lett. B94 (1980) 495.

[7] P. Langacker, S. T. Petcov, G. Steigman, and S. Toshev, On the Mikheev-Smirnov-Wolfenstein (MSW) Mechanism of Amplification of Neutrino Oscillations in Matter, Nucl. Phys. B282 (1987) 589.

[8] M. Gonzalez-Garcia, M. Maltoni, J. Salvado, and T. Schwetz, "NuFit 1.1 (2013)." http://www.nu-fit.org.

[9] M. Gonzalez-Garcia, M. Maltoni, J. Salvado, and T. Schwetz, Global fit to three neutrino mixing: critical look at present precision, JHEP 1212 (2012) 123, [arXiv:1209.3023].

[10] G. Fogli, E. Lisi, A. Marrone, D. Montanino, A. Palazzo, et al., Global analysis of neutrino masses, mixings and phases: entering the era of leptonic CP violation searches, Phys.Rev. D86 (2012) 013012, [arXiv:1205.5254].

[11] D. Forero, M. Tortola, and J. Valle, Global status of neutrino oscillation parameters after Neutrino-2012, Phys.Rev. D86 (2012) 073012, [arXiv:1205.4018].

[12] L. Wolfenstein, Neutrino Oscillations in Matter, Phys.Rev. D17 (1978) 2369-2374.

[13] S. Mikheev and A. Smirnov, Resonance Amplification of Oscillations in Matter and Spectroscopy of Solar Neutrinos, Sov.J.Nucl.Phys. 42 (1985) 913-917.

[14] E. Roulet, MSW effect with flavor changing neutrino interactions, Phys.Rev. D44 (1991) 935-938.

[15] M. Guzzo, A. Masiero, and S. Petcov, On the MSW effect with massless neutrinos and no mixing in the vacuum, Phys.Lett. B260 (1991) 154-160.

[16] V. D. Barger, R. Phillips, and K. Whisnant, Solar neutrino solutions with matter enhanced flavor changing neutral current scattering, Phys.Rev. D44 (1991) 1629-1643. 
[17] G. L. Fogli and E. Lisi, Solar Neutrino data, solar model uncertainties and solar matter enhanced neutrino oscillations, Astropart.Phys. 2 (1994) 91-100.

[18] S. Bergmann, The Solar neutrino problem in the presence of flavor changing neutrino interactions, Nucl.Phys. B515 (1998) 363-383, [hep-ph/9707398].

[19] S. Bergmann, M. Guzzo, P. de Holanda, P. Krastev, and H. Nunokawa, Status of the solution to the solar neutrino problem based on nonstandard neutrino interactions, Phys.Rev. D62 (2000) 073001, [hep-ph/0004049].

[20] M. Guzzo, H. Nunokawa, P. de Holanda, and O. Peres, On the massless 'just-so' solution to the solar neutrino problem, Phys.Rev. D64 (2001) 097301, [hep-ph/0012089].

[21] G. Fogli, E. Lisi, A. Palazzo, and A. Rotunno, Solar neutrino oscillations and indications of matter effects in the sun, Phys.Rev. D67 (2003) 073001, [hep-ph/0211414].

[22] A. Friedland, C. Lunardini, and C. Pena-Garay, Solar neutrinos as probes of neutrino matter interactions, Phys.Lett. B594 (2004) 347, [hep-ph/0402266].

[23] F. Escrihuela, O. Miranda, M. Tortola, and J. Valle, Constraining nonstandard neutrino-quark interactions with solar, reactor and accelerator data, Phys.Rev. D80 (2009) 105009, [arXiv:0907.2630].

[24] A. Bolanos, O. Miranda, A. Palazzo, M. Tortola, and J. Valle, Probing non-standard neutrino-electron interactions with solar and reactor neutrinos, Phys.Rev. D79 (2009) 113012, [arXiv:0812.4417].

[25] H. Minakata and C. Pena-Garay, Solar Neutrino Observables Sensitive to Matter Effects, arXiv: 1009.4869.

[26] A. Palazzo, Hint of non-standard MSW dynamics in solar neutrino conversion, arXiv: 1101.3875.

[27] R. Bonventre, A. LaTorre, J. Klein, G. D. O. Gann, S. Seibert, et al., Non-Standard Models, Solar Neutrinos, and Large $\theta_{13}$, arXiv:1305.5835.

[28] Y. Grossman, Nonstandard neutrino interactions and neutrino oscillation experiments, Phys.Lett. B359 (1995) 141-147, [hep-ph/9507344].

[29] M. Gonzalez-Garcia, Y. Grossman, A. Gusso, and Y. Nir, New CP violation in neutrino oscillations, Phys.Rev. D64 (2001) 096006, [hep-ph/0105159].

[30] A. Gago, M. Guzzo, H. Nunokawa, W. Teves, and R. Zukanovich Funchal, Probing flavor changing neutrino interactions using neutrino beams from a muon storage ring, Phys.Rev. D64 (2001) 073003, [hep-ph/0105196].

[31] N. Fornengo, M. Maltoni, R. Tomas, and J. Valle, Probing neutrino nonstandard interactions with atmospheric neutrino data, Phys.Rev. D65 (2002) 013010, [hep-ph/0108043].

[32] P. Huber and J. Valle, Nonstandard interactions: Atmospheric versus neutrino factory experiments, Phys.Lett. B523 (2001) 151-160, [hep-ph/0108193].

[33] T. Ota, J. Sato, and N.-a. Yamashita, Oscillation enhanced search for new interaction with neutrinos, Phys.Rev. D65 (2002) 093015, [hep-ph/0112329].

[34] P. Huber, T. Schwetz, and J. Valle, Confusing nonstandard neutrino interactions with oscillations at a neutrino factory, Phys.Rev. D66 (2002) 013006, [hep-ph/0202048].

[35] M. Campanelli and A. Romanino, Effects of new physics in neutrino oscillations in matter, Phys.Rev. D66 (2002) 113001, [hep-ph/0207350]. 
[36] T. Ota and J. Sato, Can ICARUS and OPERA give information on a new physics?, Phys.Lett. B545 (2002) 367-372, [hep-ph/0202145].

[37] M. Gonzalez-Garcia and M. Maltoni, Atmospheric neutrino oscillations and new physics, Phys.Rev. D70 (2004) 033010, [hep-ph/0404085].

[38] A. Friedland, C. Lunardini, and M. Maltoni, Atmospheric neutrinos as probes of neutrino-matter interactions, Phys.Rev. D70 (2004) 111301, [hep-ph/0408264].

[39] A. Friedland and C. Lunardini, A Test of tau neutrino interactions with atmospheric neutrinos and K2K, Phys.Rev. D72 (2005) 053009, [hep-ph/0506143].

[40] M. Blennow, T. Ohlsson, and W. Winter, Non-standard Hamiltonian effects on neutrino oscillations, Eur.Phys.J. C49 (2007) 1023-1039, [hep-ph/0508175].

[41] N. Kitazawa, H. Sugiyama, and O. Yasuda, Will MINOS see new physics?, hep-ph/0606013.

[42] A. Friedland and C. Lunardini, Two modes of searching for new neutrino interactions at MINOS, Phys.Rev. D74 (2006) 033012, [hep-ph/0606101].

[43] M. Blennow, T. Ohlsson, and J. Skrotzki, Effects of non-standard interactions in the MINOS experiment, Phys.Lett. B660 (2008) 522-528, [hep-ph/0702059].

[44] J. Kopp, M. Lindner, and T. Ota, Discovery reach for non-standard interactions in a neutrino factory, Phys.Rev. D76 (2007) 013001, [hep-ph/0702269].

[45] J. Kopp, M. Lindner, T. Ota, and J. Sato, Non-standard neutrino interactions in reactor and superbeam experiments, Phys.Rev. D77 (2008) 013007, [arXiv:0708.0152].

[46] N. Ribeiro, H. Minakata, H. Nunokawa, S. Uchinami, and R. Zukanovich-Funchal, Probing Non-Standard Neutrino Interactions with Neutrino Factories, JHEP 0712 (2007) 002, [arXiv: 0709.1980].

[47] ISS Physics Working Group Collaboration, A. Bandyopadhyay et al., Physics at a future Neutrino Factory and super-beam facility, Rept.Prog.Phys. 72 (2009) 106201, [arXiv: 0710.4947].

[48] N. C. Ribeiro, H. Nunokawa, T. Kajita, S. Nakayama, P. Ko, et al., Probing Nonstandard Neutrino Physics by Two Identical Detectors with Different Baselines, Phys.Rev. D77 (2008) 073007, [arXiv:0712.4314].

[49] A. Esteban-Pretel, J. W. Valle, and P. Huber, Can OPERA help in constraining neutrino non-standard interactions?, Phys.Lett. B668 (2008) 197-201, [arXiv:0803.1790].

[50] M. Blennow, D. Meloni, T. Ohlsson, F. Terranova, and M. Westerberg, Non-standard interactions using the OPERA experiment, Eur.Phys.J. C56 (2008) 529-536, [arXiv: 0804.2744].

[51] J. Kopp, T. Ota, and W. Winter, Neutrino factory optimization for non-standard interactions, Phys.Rev. D78 (2008) 053007, [arXiv:0804.2261].

[52] T. Ohlsson and H. Zhang, Non-Standard Interaction Effects at Reactor Neutrino Experiments, Phys.Lett. B671 (2009) 99-104, [arXiv:0809.4835].

[53] A. Palazzo and J. Valle, Confusing non-zero $\theta_{13}$ with non-standard interactions in the solar neutrino sector, Phys.Rev. D80 (2009) 091301, [arXiv:0909.1535].

[54] M. Gonzalez-Garcia, M. Maltoni, and J. Salvado, Testing matter effects in propagation of atmospheric and long-baseline neutrinos, JHEP 1105 (2011) 075, [arXiv:1103.4365]. 
[55] A. Dziewonski and D. Anderson, Preliminary reference earth model, Phys.Earth Planet.Interiors 25 (1981) 297-356.

[56] T.-K. Kuo and J. T. Pantaleone, THE SOLAR NEUTRINO PROBLEM AND THREE NEUTRINO OSCILLATIONS, Phys.Rev.Lett. 57 (1986) 1805-1808.

[57] KamLAND Collaboration, A. Gando et al., Constraints on $\theta_{13}$ from A Three-Flavor Oscillation Analysis of Reactor Antineutrinos at KamLAND, Phys.Rev. D83 (2011) 052002, [arXiv: 1009.4771].

[58] B. T. Cleveland et al., Measurement of the solar electron neutrino flux with the Homestake chlorine detector, Astrophys. J. 496 (1998) 505-526.

[59] F. Kaether, W. Hampel, G. Heusser, J. Kiko, and T. Kirsten, Reanalysis of the GALLEX solar neutrino flux and source experiments, Phys. Lett. B685 (2010) 47-54, [arXiv: 1001.2731].

[60] SAGE Collaboration, J. N. Abdurashitov et al., Measurement of the solar neutrino capture rate with gallium metal. III: Results for the 2002-2007 data-taking period, Phys. Rev. C80 (2009) 015807, [arXiv:0901.2200].

[61] Super-Kamiokande Collaboration, J. Hosaka et al., Solar neutrino measurements in Super-Kamiokande-I, Phys. Rev. D73 (2006) 112001, [hep-ex/0508053].

[62] Super-Kamiokande Collaboration, J. Cravens et al., Solar neutrino measurements in Super-Kamiokande-II, Phys.Rev. D78 (2008) 032002, [arXiv:0803.4312].

[63] Super-Kamiokande Collaboration, K. Abe et al., Solar neutrino results in Super-Kamiokande-III, Phys.Rev. D83 (2011) 052010, [arXiv:1010.0118].

[64] M. Smy, "Super-Kamiokande's Solar $\nu$ Results." Talk given at the XXV International Conference on Neutrino Physics, Kyoto, Japan, June 3-9, 2012.

[65] Borexino Collaboration, G. Bellini et al., Precision measurement of the 7Be solar neutrino interaction rate in Borexino, Phys.Rev.Lett. 107 (2011) 141302, [arXiv:1104.1816].

[66] Borexino Collaboration, G. Bellini et al., Measurement of the solar $8 B$ neutrino rate with a liquid scintillator target and $3 \mathrm{MeV}$ energy threshold in the Borexino detector, Phys. Rev. D82 (2010) 033006, [arXiv:0808.2868].

[67] SNO Collaboration, B. Aharmim et al., Measurement of the nu/e and total B-8 solar neutrino fluxes with the Sudbury Neutrino Observatory phase I data set, Phys. Rev. C75 (2007) 045502, [nucl-ex/0610020].

[68] SNO Collaboration, B. Aharmim et al., Electron energy spectra, fluxes, and day-night asymmetries of B-8 solar neutrinos from the 391-day salt phase SNO data set, Phys. Rev. C72 (2005) 055502, [nucl-ex/0502021].

[69] SNO Collaboration, B. Aharmim et al., An Independent Measurement of the Total Active 8B Solar Neutrino Flux Using an Array of 3He Proportional Counters at the Sudbury Neutrino Observatory, Phys. Rev. Lett. 101 (2008) 111301, [arXiv:0806. 0989].

[70] SNO Collaboration, B. Aharmim et al., Combined Analysis of all Three Phases of Solar Neutrino Data from the Sudbury Neutrino Observatory, arXiv:1109.0763.

[71] J. N. Bahcall, A. M. Serenelli, and S. Basu, New solar opacities, abundances, helioseismology, and neutrino fluxes, Astrophys.J. 621 (2005) L85-L88, [astro-ph/0412440]. 
[72] C. Pena-Garay and A. Serenelli, Solar neutrinos and the solar composition problem, arXiv:0811.2424.

[73] O. Miranda, M. Tortola, and J. Valle, Are solar neutrino oscillations robust?, JHEP 0610 (2006) 008, [hep-ph/0406280]

[74] L. Pik, "Study of the neutrino mass hierarchy with the atmospheric neutrino data observed in super-kamiokande.." Ph.D. Thesis, 2012.

[75] Super-Kamiokande Collaboration, R. Wendell et al., Atmospheric neutrino oscillation analysis with sub-leading effects in Super-Kamiokande I, II, and III, Phys. Rev. D81 (2010) 092004, [arXiv: 1002.3471].

[76] MINOS Collaboration, P. Adamson et al., Measurement of Neutrino and Antineutrino Oscillations Using Beam and Atmospheric Data in MINOS, arXiv:1304.6335.

[77] MINOS Collaboration, P. Adamson et al., Electron neutrino and antineutrino appearance in the full MINOS data sample, Phys.Rev.Lett. (2013) [arXiv:1301.4581].

[78] M. Ikeda. Talk given at the Conference Reecontres de Moriond EW 2013, La Thuile, Italy, March 2-9, 2013.

[79] CHOOZ Collaboration, M. Apollonio et al., Limits on Neutrino Oscillations from the CHOOZ Experiment, Phys. Lett. B466 (1999) 415-430, [hep-ex/9907037].

[80] Palo Verde Collaboration, A. Piepke, Final results from the Palo Verde neutrino oscillation experiment, Prog.Part.Nucl.Phys. 48 (2002) 113-121.

[81] Double Chooz Collaboration, Y. Abe et al., Reactor electron antineutrino disappearance in the Double Chooz experiment, Phys.Rev. D86 (2012) 052008, [arXiv:1207.6632].

[82] Daya Bay Collaboration, F. An et al., Improved Measurement of Electron Antineutrino Disappearance at Daya Bay, Chin. Phys. C37 (2013) 011001, [arXiv:1210.6327].

[83] S.-H. Seo. Talk given at the XV International Workshop on Neutrino Telescopes, Venice, Italy, March 11-15,2013.

[84] T. Schwetz, M. Tortola, and J. Valle, Global neutrino data and recent reactor fluxes: status of three-flavour oscillation parameters, New J.Phys. 13 (2011) 063004, [arXiv:1103.0734].

[85] Y. Declais, H. de Kerret, B. Lefievre, M. Obolensky, A. Etenko, et al., Study of reactor anti-neutrino interaction with proton at Bugey nuclear power plant, Phys.Lett. B338 (1994) 383-389.

[86] A. Kuvshinnikov, L. Mikaelyan, S. Nikolaev, M. Skorokhvatov, and A. Etenko, Measuring the $\bar{\nu}_{e}+p \rightarrow n+e^{+}$cross-section and beta decay axial constant in a new experiment at Rovno NPP reactor. (In Russian), JETP Lett. 54 (1991) 253-257.

[87] Y. Declais, J. Favier, A. Metref, H. Pessard, B. Achkar, et al., Search for neutrino oscillations at 15-meters, 40-meters, and 95-meters from a nuclear power reactor at Bugey, Nucl.Phys. B434 (1995) 503-534.

[88] G. Vidyakin, V. Vyrodov, I. Gurevich, Y. Kozlov, V. Martemyanov, et al., Detection of anti-neutrinos in the flux from two reactors, Sov.Phys.JETP 66 (1987) 243-247.

[89] G. Vidyakin, V. Vyrodov, Y. Kozlov, A. Martemyanov, V. Martemyanov, et al., Limitations on the characteristics of neutrino oscillations, JETP Lett. 59 (1994) 390-393.

[90] H. Kwon, F. Boehm, A. Hahn, H. Henrikson, J. Vuilleumier, et al., Search for neutrino oscillations at a fission reactor, Phys.Rev. D24 (1981) 1097-1111. 
[91] CALTECH-SIN-TUM Collaboration, G. Zacek et al., Neutrino Oscillation Experiments at the Gosgen Nuclear Power Reactor, Phys.Rev. D34 (1986) 2621-2636.

[92] Z. D. Greenwood et al., Results of a two position reactor neutrino oscillation experiment, Phys. Rev. D53 (1996) 6054-6064.

[93] A. Afonin, S. Ketov, V. Kopeikin, L. Mikaelyan, M. Skorokhvatov, et al., A study of the reaction $\bar{\nu}_{e}+p \rightarrow e^{+}+n$ on a nuclear reactor, Sov.Phys.JETP 67 (1988) 213-221.

[94] CHARM Collaboration, J. Dorenbosch et al., Experimental verification of the universality of electron-neutrino and muon-neutrino coupling to the neutral weak current, Phys.Lett. B180 (1986) 303.

[95] CHARM Collaboration, J. Allaby et al., A Precise Determination of the Electroweak Mixing Angle from Semileptonic Neutrino Scattering, Z.Phys. C36 (1987) 611.

[96] A. Blondel, P. Bockmann, H. Burkhardt, F. Dydak, A. Grant, et al., Electroweak parameters from a high statistics neutrino nucleon scattering experiment, Z.Phys. C45 (1990) 361-379.

[97] NuTeV Collaboration, G. Zeller et al., A Precise determination of electroweak parameters in neutrino nucleon scattering, Phys.Rev.Lett. 88 (2002) 091802, [hep-ex/0110059].

[98] S. Davidson, C. Pena-Garay, N. Rius, and A. Santamaria, Present and future bounds on nonstandard neutrino interactions, JHEP 0303 (2003) 011, [hep-ph/0302093].

[99] C. Biggio, M. Blennow, and E. Fernandez-Martinez, General bounds on non-standard neutrino interactions, JHEP 0908 (2009) 090, [arXiv:0907.0097]. 